ESAIM: COCV 21 (2015) 583-601

DOI: $10.1051 / \mathrm{cocv} / 2014039$
ESAIM: Control, Optimisation and Calculus of Variations

www.esaim-cocv.org

\title{
STABILITY AND BOUNDARY CONTROLLABILITY OF A LINEARIZED MODEL OF FLOW IN AN ELASTIC TUBE
}

\author{
Gilbert Peralta ${ }^{1,2}$ And Georg Propst ${ }^{2}$
}

\begin{abstract}
We consider a model describing the flow of a fluid inside an elastic tube that is connected to two tanks. We study the linearized system through semigroup theory. Controlling the pressures in the tanks renders a hyperbolic PDE with boundary control. The linearization induces a one-dimensional linear manifold of equilibria; when those are factored out, the corresponding semigroup is exponentially stable. The location of the eigenvalues in dependence on the viscosity is discussed. Exact boundary controllability of the system is achieved by the Riesz basis approach including generalized eigenvectors. A minimal time for controllability is given. The corresponding result for internal distributed control is stated.
\end{abstract}

Mathematics Subject Classification. 35L50, 47D03, 93C20.

Received March 19, 2013.

Published online March 19, 2015.

\section{THE MODEL AND ITS LINEARIZED VERSION}

Consider an elastic cylindrical tube of length $\ell$ filled with an incompressible liquid and each end of the tube is linked to one of two tanks, both with horizontal cross section $A_{T}$ (see Fig. 1). Let $u(t, x), A(t, x)$ and $p(t, x)$ denote the fluid velocity, the vertical cross section of the tube and the pressure in the tube, respectively, at time $t \geq 0$ and position $x \in[0, \ell]$. The rest cross section of the tube is denoted by $A_{0}=\pi r_{0}^{2}$, where $r_{0}$ is the inner rest radius, and the ambient pressure is denoted by $p_{a}$. According to Laplace's law for cylinders and Hooke's law for the radial tension in the tube material, the equation of state, as in Rath and Teipel [18], is given by

$$
A(t, x)=A_{0}\left(1+\frac{r_{0}}{s E}\left(p(t, x)-p_{a}\right)\right)^{2},
$$

where $E$ is the Young's modulus and $s$ is the thickness of the tube material. We note that longitudinal tension is not taken into account in this model, i.e., the radial tension at $x$ is independent of the neighborhood of $x$.

Keywords and phrases. Flow in elastic tube, semigroup, exponential stability, boundary control system, exact controllability, Riesz basis.

1 Department of Mathematics and Computer Science, University of the Philippines Baguio, Governor Pack Road, 2600 Baguio City, Philippines. grperalta@upb.edu.ph

2 Institut für Mathematik und Wissenschaftliches Rechnen, NAWI Graz, Karl-Franzens-Universität Graz, Heinrichstraße 36, 8010 Graz, Austria. georg.propst@uni-graz.at 


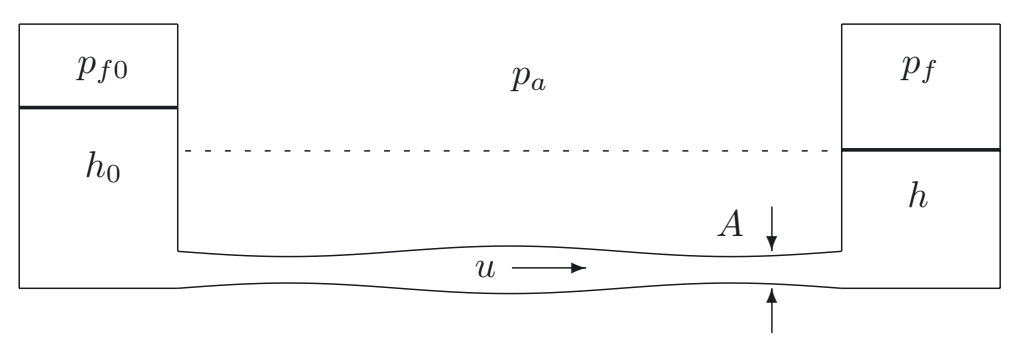

Figure 1. An elastic tube connected to two tanks.

Using Euler's continuity equation and the law of balance of momentum, the cross sectional area and the fluid velocity are given by, as in Ottesen [17],

$$
\begin{cases}\frac{\partial A}{\partial t}=-u \frac{\partial A}{\partial x}-A \frac{\partial u}{\partial x}, & t>0,0<x<\ell, \\ \frac{\partial u}{\partial t}=-u \frac{\partial u}{\partial x}-\frac{s E}{2 \rho r_{0}}\left(A_{0} A\right)^{-1 / 2} \frac{\partial A}{\partial x}-\frac{8 \pi \mu}{\rho A_{0}} u, & t>0,0<x<\ell,\end{cases}
$$

where $\rho$ and $\mu$ are the constant density and the viscosity of the fluid respectively. The last component of the right hand side of the balance equation in (1.2) represents friction, according to the model of Hagen-Poiseuille.

We are interested in the well-posedness, stability and controllability of the linearized model. In this work, we consider forcing pressures that are applied on top of the tanks. The pressure at the end of the tube is then the sum of the ambient pressure, the hydrostatic pressure and the forcing pressure. This gives the boundary conditions

$$
\begin{aligned}
& p(t, 0)=p_{a}+\rho g h_{0}(t)+p_{f 0}(t), \\
& p(t, \ell)=p_{a}+\rho g h(t)+p_{f}(t),
\end{aligned}
$$

where $g$ is the gravitational constant and $p_{f 0}$ and $p_{f}$ are the forcing pressures applied to the left and right tank at $x=0$ and $x=\ell$, respectively. In terms of the cross sectional area we can see from the equation of state (1.1) that these boundary conditions become

$$
\begin{array}{ll}
A(t, 0)=A_{0}\left(1+\frac{r_{0}}{s E}\left(\rho g h_{0}(t)+p_{f 0}(t)\right)\right)^{2}, & t>0, \\
A(t, \ell)=A_{0}\left(1+\frac{r_{0}}{s E}\left(\rho g h(t)+p_{f}(t)\right)\right)^{2}, & t>0,
\end{array}
$$

Note that the tube ends' cross sections are free to adjust to the inner pressure that is prescribed by the pressures at the bottom of the tanks.

The level heights $h_{0}$ and $h$ in the tanks are state components starting at some given initial height $h_{0}^{0}$ and $h^{0}$, respectively, and are coupled to the cross section and fluid velocity by

$$
\left\{\begin{aligned}
\frac{\mathrm{d} h_{0}}{\mathrm{~d} t} & =-\frac{1}{A_{T}} A(t, 0) u(t, 0), \quad t>0, \\
\frac{\mathrm{d} h}{\mathrm{~d} t} & =\frac{1}{A_{T}} A(t, \ell) u(t, \ell), \quad t>0,
\end{aligned}\right.
$$

See $[2,17,18]$ for similar models. System of equations similar to $(1.2)$ are also used in multiscale blood flow models, for example, in $[5,19]$.

Let us determine the equilibria of the above system when $p_{f 0}$ and $p_{f}$ do not depend on $t$. Setting the derivative with respect to time to zero in (1.2), the first equation will give $\partial(A u) / \partial x=0$ and so $A u$ is constant on $[0, \ell]$. 
However, the third and fourth equation will give $A(t, 0) u(t, 0)=A(t, \ell) u(t, \ell)=0$ and assuming that $A$ remains positive for all $t \geq 0$ it follows that $u$ must be identically zero on $[0, \ell]$. Using this information in the second equation we obtain that $\partial A / \partial x=0$ and so $A$ must be constant on the domain, say $A=A_{e}$. Because $\mathrm{d} h_{0} / \mathrm{d} t=0$ and $\mathrm{d} h / \mathrm{d} t=0$ then $h_{0}=h_{0 e}$ and $h=h_{e}$ for some constants $h_{0 e}$ and $h_{e}$. Thus we have

$$
A_{e}=A_{0}\left(1+\frac{r_{0}}{s E}\left(\rho g h_{0 e}+p_{f 0}\right)\right)^{2}=A_{0}\left(1+\frac{r_{0}}{s E}\left(\rho g h_{e}+p_{f}\right)\right)^{2} .
$$

and it follows that $h_{e}-h_{0 e}=\frac{1}{\rho g}\left(p_{f 0}-p_{f}\right)$. We ignore the other possibility $h_{0 e}+h_{e}=-\frac{1}{\rho r_{0} g}\left(2 s E+r_{0} p_{f 0}+r_{0} p_{f}\right)$ since we are interested in the case where the level heights in the tanks are both positive. If $p_{f 0}=p_{f}$ then the former equality coincides with the fact that the level heights in the two tanks must be the same. Note also that this is true even when the two tanks have different horizontal cross sections. If $V$ denotes the volume of fluid in the tube and in the tanks, then $V=A_{e} \ell+A_{T}\left(h_{0 e}+h_{e}\right)$. Therefore $p_{f 0}, p_{f}$ and $V$ uniquely determine the equilibrium point. Furthermore, it is easy to see that we can choose $p_{f 0}$ and $p_{f}$ such that $h_{0 e}$ and $h_{e}$ are both positive.

To linearize the above system about the equilibrium point $z_{e}=\left(A_{e}, u_{e},\left(A_{e}\right)_{x},\left(u_{e}\right)_{x}, h_{0 e}, h_{e}\right)$, where $u_{e}=$ $\left(A_{e}\right)_{x}=\left(u_{e}\right)_{x}=0$, we use the Taylor series expansions of the right hand sides of (1.2), (1.5), (1.6) and (1.7) about the equilibrium $z_{e}$ and neglect the terms of order higher than one. From these equations we let $A=A_{e}+\tilde{A}$, $u=\tilde{u}, h_{0}=h_{0 e}+\tilde{h}_{0}$ and $h=h_{e}+\tilde{h}$, which are the small deviations from the equilibrium, to obtain the linearized system

$$
\begin{cases}\frac{\partial \tilde{A}}{\partial t}=-A_{e} \frac{\partial \tilde{u}}{\partial x}, & t>0,0<x<\ell, \\ \frac{\partial \tilde{u}}{\partial t}=-\alpha \frac{\partial \tilde{A}}{\partial x}-\beta \tilde{u}, & t>0,0<x<\ell, \\ \frac{\mathrm{d} \tilde{h}_{0}}{\mathrm{~d} t}=-\frac{A_{e}}{A_{T}} \tilde{u}(t, 0), & t>0, \\ \frac{\mathrm{d} \tilde{h}}{\mathrm{~d} t}=\frac{A_{e}}{A_{T}} \tilde{u}(t, \ell), & t>0,\end{cases}
$$

with boundary conditions

$$
\tilde{A}(t, 0)=\gamma \tilde{h}_{0}(t), \quad \tilde{A}(t, \ell)=\gamma \tilde{h}(t), \quad t>0,
$$

and initial conditions

$$
\left\{\begin{array}{lrl}
\tilde{A}(0, x)=\tilde{A}^{0}(x), & \tilde{u}(0, x)=\tilde{u}^{0}(x), 0 \leq x \leq \ell, \\
\tilde{h}_{0}(0)=\tilde{h}_{0}^{0}, & \tilde{h}(0)=\tilde{h}^{0}
\end{array}\right.
$$

In the above system we used the following notations

$$
\begin{aligned}
& \alpha=\frac{s E}{2 \rho r_{0} \sqrt{A_{0} A_{e}}}, \quad \quad \beta=\frac{8 \pi \mu}{\rho A_{0}}, \\
& \gamma=\frac{2 \rho A_{0} g r_{0}}{s E}\left(1+\frac{r_{0}}{s E}\left(\rho g h_{0 e}+p_{f 0}\right)\right)=\frac{2 \rho A_{0} g r_{0}}{s E}\left(1+\frac{r_{0}}{s E}\left(\rho g h_{e}+p_{f}\right)\right),
\end{aligned}
$$

since, for the linearization, we assume that $p_{f 0}$ and $p_{f}$ are constants. We remark that all the parameters $r_{0}, s, A_{0}, A_{e}, E$ are positive while $\mu$ is nonnegative. As a result, $\alpha>0$ and $\beta \geq 0$. The constants $p_{f 0}$ and $p_{f}$ can also be chosen to be small, so that $\gamma>0$. The resulting linear system is the coupling of PDEs in one space dimension with ODEs and sometimes such systems are referred to as hybrid systems. By differentiation, a second order linear model, which is a wave equation with viscous damping and Robin boundary conditions, was formulated and discussed by Bredow [23]. 
In Section 2, we show that the operator corresponding to the abstract formulation of the linearized model generates a strongly continuous semigroup of contractions on the whole state space. We show in Section 3 that under the presence of friction there are no eigenvalues of the operator on the imaginary axis other than the origin. The semigroup is then exponentially stable if it is restricted to the orthogonal complement of the eigenspace corresponding to the eigenvalue 0 . This eigenspace can be viewed as a space of equilibria of the linearized model.

A boundary control system is considered in Section 4. For more details on boundary control systems, we refer to the book of Tucsnak and Weiss ([22], Chap. 10) and the references therein. The main idea is to reformulate an abstract initial-boundary value problem as a pure initial-value problem in an extended space. We use the Riesz basis approach to prove the exact controllability of the system. To do this, we modify the arguments in ([22], Prop. 8.1.3) which work in orthonormal bases. Unlike orthonormal bases, Riesz bases in general do not satisfy the property of being orthogonal. The Riesz basis approach has been successfully used by Guo and collaborators to prove the stability of certain beam equations [6-9]. The basic idea of Riesz basis generation in these papers is the application of a result similar to Bari's Theorem ([26], Thm. 15), i.e., to prove that a sequence of generalized eigenvectors is quadratically close to a given Riesz basis. Unlike beam equations, which have increasing spectral gap (distance between consecutive eigenvalues), wave equations have an asymptotically constant spectral gap. A refinement of the Riesz basis generation theorem of Guo ([6], Thm. 6.3), was given recently by Xu and Weiss ([25], Thm. 2.4). The latter result will be used in proving that the infinitesimal generator is Riesz spectral, i.e., has a Riesz basis consisting of generalized eigenvectors. Furthermore, the uniform gap property of the spectrum also plays an important role for the application of Ingham's theorem, which is used in the proof of exact controllability.

A minimal time of controllability for single input controls will be given. However, Ingham's Theorem will not be applicable in this problem and we need to use other perturbation results in non-harmonic Fourier analysis. In order to solve this, we separate the lower and higher frequencies and replace the non-harmonic Fourier basis elements corresponding to the lower frequencies by some harmonic ones. With this in hand, the problem will be solved by applying a generalized Kadec's $\frac{1}{4}$-Theorem (see e.g. [26], Cor. 2, p. 196).

Finally, we will state an analogous result for distributed control which is done, e.g. in $[2,18,23]$.

\section{WELL-POSEDNESS OF THE LINEAR SYSTEM}

In this section we prove the well-posedness of the linear system (1.8)-(1.10). For convenience, we will denote the state variables and the initial conditions for the linearized system without the tildas. Our approach utilizes the theory of strongly continuous semigroups. We will recast the system as a differential equation in an infinitedimensional state space. Consider the Hilbert space $\mathcal{X}=L^{2}((0, \ell), \mathbb{C})^{2} \times \mathbb{C}^{2}$ equipped with the inner product

$$
\left\langle\left(\varphi_{1}, \psi_{1}, a_{1}, b_{1}\right),\left(\varphi_{2}, \psi_{2}, a_{2}, b_{2}\right)\right\rangle_{\mathcal{X}}=\frac{1}{A_{e}}\left\langle\varphi_{1}, \varphi_{2}\right\rangle_{L^{2}}+\frac{1}{\alpha}\left\langle\psi_{1}, \psi_{2}\right\rangle_{L^{2}}+\frac{\gamma A_{T}}{A_{e}}\left(a_{1} \overline{a_{2}}+b_{1} \overline{b_{2}}\right) .
$$

Notice that the norm induced by the above inner product is equivalent to the usual product norm of $\mathcal{X}$.

Define the linear operator $\mathcal{A}: \mathcal{D}(\mathcal{A}) \rightarrow \mathcal{X}$ with domain $\mathcal{D}(\mathcal{A})=\left\{\left(A, u, h_{0}, h\right) \in \mathcal{X}: A, u \in H^{1}(0, \ell), A(0)=\right.$ $\left.\gamma h_{0}, A(\ell)=\gamma h\right\}$ by

$$
\mathcal{A}\left(\begin{array}{c}
A \\
u \\
h_{0} \\
h
\end{array}\right)=\left(\begin{array}{c}
-A_{e} u_{x} \\
-\alpha A_{x}-\beta u \\
-\frac{A_{e}}{A_{T}} u(0) \\
\frac{A_{e}}{A_{T}} u(\ell)
\end{array}\right) .
$$


Observe that the last two components of the state appear only in the domain of $\mathcal{A}$. The coupled system (1.8) can now be phrased as an abstract Cauchy problem

$$
(\mathrm{ACP}) \quad\left\{\begin{aligned}
\frac{\mathrm{d}}{\mathrm{d} t}\left(A, u, h_{0}, h\right)(t) & =\mathcal{A}\left(A, u, h_{0}, h\right)(t), \quad t>0, \\
\left(A, u, h_{0}, h\right)(0) & =\left(A^{0}, u^{0}, h_{0}^{0}, h^{0}\right)
\end{aligned}\right.
$$

on the state space $\mathcal{X}$.

There are several ways to prove the well-posedness of (ACP). One possible approach is to split the PDE and the ODE. The PDE can be considered as a port-Hamiltonian system and hence it is well-posed ([11], Chap. 13), and the ODE, being finite-dimensional, is also well-posed. Then one shows that the feedback interconnection of the two systems is well-posed ([20], Sect. 7.2).

However, the approach presented here is based on the following lemma. It is a recap of the proof of Theorem 3 in [3]. $X^{\prime}$ denotes the dual space of $X$ and $\mathcal{L}(X, Y)$ is the space of bounded linear operators from $X$ to $Y$.

Lemma 2.1 (Lax-Milgram-Fredholm). Let $V$ and $H$ be Hilbert spaces such that the embedding $V \subset H$ is compact and dense. Suppose that $a_{1}: V \times V \rightarrow \mathbb{C}$ and $a_{2}: H \times H \rightarrow \mathbb{C}$ are two bounded sesquilinear forms such that $a_{1}$ is $V$-coercive and $F: V \rightarrow \mathbb{C}$ is a continuous conjugate linear form. The equation

$$
a_{1}(u, v)+a_{2}(u, v)=F(v), \quad \forall v \in V,
$$

has either a unique solution $u \in V$ for all $F \in V^{\prime}$ or has a nontrivial solution for $F=0$.

Proof. Since $a_{1}$ is bounded, the operator $T: V \rightarrow V^{\prime}$ defined by $\langle T \varphi, \psi\rangle_{V^{\prime} \times V}=a_{1}(\varphi, \psi)$ for all $\varphi, \psi \in V$ is bounded. Furthermore, by the Lax-Milgram Lemma we have $T^{-1} \in \mathcal{L}\left(V^{\prime}, V\right)$. Define $S: H \rightarrow V^{\prime}$ by $\langle S \varphi, \psi\rangle_{V^{\prime} \times V}=a_{2}(\varphi, \psi)$. Since for each $\varphi \in H$

$$
\|S \varphi\|_{V^{\prime}}=\sup _{\|\psi\|_{V}=1}\left|\langle S \varphi, \psi\rangle_{V^{\prime} \times V}\right| \leq \sup _{\|\psi\|_{V}=1} C\|\varphi\|_{H}\|\psi\|_{H} \leq \tilde{C}\|\varphi\|_{H}
$$

it holds that $S \in \mathcal{L}\left(H, V^{\prime}\right)$ and in particular $S \in \mathcal{L}\left(V, V^{\prime}\right)$ is compact. The equation (2.1) is equivalent to $\left(1+T^{-1} S\right) v=T^{-1} F$ in $V$. Since $T^{-1} S$ is compact the Fredholm alternative implies that either $-1 \in \rho\left(T^{-1} S\right)$ or $-1 \in \sigma_{p}\left(T^{-1} S\right)$, where $\rho(A)$ and $\sigma_{p}(A)$ denote the resolvent set and point spectrum of a closed operator $A$.

Theorem 2.2. The operator $\mathcal{A}$ generates a strongly continuous semigroup of contractions on $\mathcal{X}$, and in particular, for every $\left(A^{0}, u^{0}, h_{0}^{0}, h^{0}\right) \in \mathcal{D}(\mathcal{A})$ there exist unique functions $A, u \in C^{1}\left([0, \infty), L^{2}(0, \ell)\right)$ and $h_{0}, h \in C^{1}[0, \infty)$ such that $(\mathrm{ACP})$ is satisfied.

Proof. We will use the Lumer-Phillips Theorem in reflexive Banach spaces (see e.g. [4], Cor. III.3.20). Integrating by parts and using the boundary conditions we have

$$
\left\langle\mathcal{A}\left(A, u, h_{0}, h\right),\left(A, u, h_{0}, h\right)\right\rangle_{\mathcal{X}}=-\frac{\beta}{\alpha}\|u\|_{L^{2}}^{2}+2 i \Im\left\langle u, A_{x}\right\rangle_{L^{2}}
$$

for all $\left(A, u, h_{0}, h\right) \in \mathcal{D}(\mathcal{A})$. Taking the real part shows that $\mathcal{A}$ is dissipative. Next we are going to show the range condition. Fix $\lambda>0$ and $\left(B, v, g_{0}, g\right) \in \mathcal{X}$ and define $a_{1}: H^{1}(0, \ell) \times H^{1}(0, \ell) \rightarrow \mathbb{C}, a_{2}: L^{2}(0, \ell) \times L^{2}(0, \ell) \rightarrow \mathbb{C}$ and $F: H^{1}(0, \ell) \rightarrow \mathbb{C}$ by

$$
\begin{aligned}
a_{1}(\varphi, \psi) & =\frac{\alpha \gamma A_{e}}{A_{T}(\lambda+\beta)}\langle\varphi, \psi\rangle_{H^{1}}+\lambda \varphi(0) \overline{\psi(0)}+\lambda \varphi(\ell) \overline{\psi(\ell)} \\
a_{2}(\varphi, \psi) & =\frac{\gamma}{A_{T}}\left(\lambda-\frac{\alpha A_{e}}{\lambda+\beta}\right)\langle\varphi, \psi\rangle_{L^{2}} \\
F(\psi) & =\frac{\gamma}{A_{T}} \int_{0}^{\ell} B(x) \overline{\psi(x)} \mathrm{d} x+\frac{\gamma A_{e}}{A_{T}(\lambda+\beta)} \int_{0}^{\ell} v(x) \overline{\psi_{x}(x)} \mathrm{d} x+\gamma g_{0} \overline{\psi(0)}+\gamma g \overline{\psi(\ell)},
\end{aligned}
$$


respectively. Note that the sesquilinear forms $a_{1}, a_{2}$ and the conjugate linear form $F$ satisfy the conditions of Lemma 2.1. It can be shown (see the Appendix for details) that the equation

$$
(\lambda I-\mathcal{A})\left(A, u, h_{0}, h\right)=\left(B, v, g_{0}, g\right)
$$

has a solution $\left(A, u, h_{0}, h\right) \in \mathcal{D}(\mathcal{A})$ if and only if there is an $A \in H^{1}(0, \ell)$ that satisfies

$$
a_{1}(A, \psi)+a_{2}(A, \psi)=F(\psi), \quad \forall \psi \in H^{1}(0, \ell)
$$

We prove that the second case in Lemma 2.1 does not hold. Suppose that $a_{1}(A, \psi)+a_{2}(A, \psi)=0$ for all $\psi \in H^{1}(0, \ell)$. This condition is equivalent to the system $(2.2)$ with $\left(B, v, g_{0}, g\right)=0$. From the first equation we get $A=-\frac{A_{e}}{\lambda} u_{x}$. The rest of the equations will give us

$$
\begin{aligned}
\langle A, A\rangle_{L^{2}} & =-\frac{A_{e}}{\lambda} \int_{0}^{\ell} u_{x}(x) \overline{A(x)} \mathrm{d} x \\
& =-\frac{A_{e}}{\lambda}(u(\ell) \overline{A(\ell)}-u(0) \overline{A(0)})+\frac{A_{e}}{\lambda} \int_{0}^{\ell} u(x) \overline{A_{x}(x)} \mathrm{d} x \\
& =-\frac{A_{T}}{\gamma}\left(|A(\ell)|^{2}+|A(0)|^{2}\right)-\frac{\alpha A_{e}}{\lambda(\lambda+\beta)} \int_{0}^{\ell}\left|A_{x}(x)\right|^{2} \mathrm{~d} x \leq 0 .
\end{aligned}
$$

Hence $A=0$. This proves the range condition and hence completes the proof of the theorem.

Remark 2.3. If the initial data $A^{0}, u^{0} \in H^{2}(0, \ell)$ and $h_{0}^{0}, h_{0} \in \mathbb{C}$ satisfy the compatibility conditions $A^{0}(0)=$ $\gamma h_{0}^{0}, A(\ell)=\gamma h^{0}, A_{T} u_{x}^{0}(0)=\gamma u^{0}(0)$ and $-A_{T} u_{x}^{0}(\ell)=\gamma u^{0}(\ell)$ then we have a classical solution $A, u \in C^{1}([0, \infty) \times$ $[0, \ell])$ and $h_{0}, h \in C^{2}[0, \infty)$.

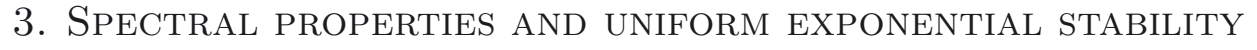

At this point, we already know that $\sigma(\mathcal{A}) \subset\{z \in \mathbb{C}: \Re z \leq 0\}$ since $\mathcal{A}$ generates a contractive $\mathcal{C}_{0}$-semigroup on $\mathcal{X}$. Furthermore, the adjoint operator $\mathcal{A}^{*}$ also generates a contraction $\mathcal{C}_{0}$-semigroup, which is the adjoint semigroup, in other words, $\left(\mathrm{e}^{\mathcal{A} t}\right)^{*}=\mathrm{e}^{\mathcal{A}^{*} t}$ for all $t \geq 0$.

Let us determine the $\mathcal{X}$-adjoint of $\mathcal{A}$. Define $\tilde{\mathcal{A}}: \mathcal{D}(\mathcal{A}) \rightarrow \mathcal{X}$ by

$$
\tilde{\mathcal{A}}\left(\begin{array}{c}
B \\
v \\
g_{0} \\
g
\end{array}\right)=\left(\begin{array}{c}
A_{e} v_{x} \\
\alpha B_{x}-\beta v \\
\frac{A_{e}}{A_{T}} v(0) \\
-\frac{A_{e}}{A_{T}} v(\ell)
\end{array}\right) .
$$

For each $\left(A, u, h_{0}, h\right),\left(B, v, g_{0}, g\right) \in \mathcal{D}(\mathcal{A})$, a straight forward computation yields

$$
\left\langle\mathcal{A}\left(A, u, h_{0}, h\right),\left(B, v, g_{0}, g\right)\right\rangle_{\mathcal{X}}=\left\langle\left(A, u, h_{0}, h\right), \tilde{\mathcal{A}}\left(B, v, g_{0}, g\right)\right\rangle_{\mathcal{X}}
$$

which implies that $\left(B, v, g_{0}, g\right) \in \mathcal{D}\left(\mathcal{A}^{*}\right)$, and this proves that $\mathcal{A}^{*}$ is an extension of $\tilde{\mathcal{A}}$. Using a similar argument as in the proof of Theorem 2.2, we can also show that $\tilde{\mathcal{A}}$ generates a $\mathcal{C}_{0}$-semigroup of contractions on $\mathcal{X}$, and hence $(0, \infty) \subset \rho\left(\mathcal{A}^{*}\right) \cap \rho(\tilde{\mathcal{A}})$. Applying ([16], Lem. 1.6.14), we can see that $\mathcal{A}^{*}=\tilde{\mathcal{A}}$ and in particular $\mathcal{D}\left(\mathcal{A}^{*}\right)=\mathcal{D}(\tilde{\mathcal{A}})=\mathcal{D}(\mathcal{A})$.

In the absence of friction, i.e. $\beta=0$, we have $\mathcal{A}^{*}=-\mathcal{A}$ and so $\mathcal{A}$ is skew-adjoint and from Stone's Theorem (see e.g. [4], Thm. 3.24) the operator $\mathcal{A}$ generates a unitary $\mathcal{C}_{0}$-group. This will be used in the succeeding section. The operators $\mathcal{A}$ and $\mathcal{A}^{*}$ also generate $\mathcal{C}_{0}$-groups even for $\beta>0$. To see this, let us define $\mathcal{C} \in \mathcal{L}(\mathcal{X})$ by $\mathcal{C}\left(A, u, h_{0}, h\right)=(0, u, 0,0)$. Then $-\mathcal{A}=\mathcal{A}^{*}+2 \beta \mathcal{C}$ and $-\mathcal{A}$ generates a $\mathcal{C}_{0}$-semigroup satisfying $\left\|\mathrm{e}^{-\mathcal{A} t}\right\| \leq \mathrm{e}^{2 \beta t}$ for all $t \geq 0$ (see, e.g. [4], Thm. III.1.3). From Theorem 2.2 and ([4], p. 79), $\mathcal{A}$ generates a $\mathcal{C}_{0}$-group on $\mathcal{X}$ satisfying 
$\left\|\mathrm{e}^{\mathcal{A} t}\right\| \leq \mathrm{e}^{2 \beta|t|}$ for all $t \in \mathbb{R}$. The case of $\mathcal{A}^{*}$ is analogous. Tight decay rates will be given after we have described the spectra of the generators.

The operators $\mathcal{A}$ and $\mathcal{A}^{*}$ have compact resolvents and therefore their spectra consist of eigenvalues only. This is a consequence of the compactness of the embedding $H^{1}(0, \ell) \hookrightarrow L^{2}(0, \ell)$. We can now characterize the spectrum of $\mathcal{A}$ and its adjoint. Due to the differential boundary conditions, namely the third and fourth lines in (1.8), the eigenvalues appear on the boundary conditions of a two-point boundary value problem, see (3.5) for instance. To describe the spectrum of the differential operator for $\beta \geq 0$, we first describe the special case where $\beta=0$ and use this to investigate for the case $\beta>0$. First, we state a lemma needed for the asymptotic description of the eigenvalues.

Lemma 3.1. Let $a, b, c>0$ and $H(x)=x \cos a x-\left(b x^{2}-c\right) \sin a x$ and let $\left(\mu_{n}\right)_{n \in \mathbb{N}}$, listed in strictly increasing order, be the positive zeros of $H$. Then $\mu_{n}=\frac{(n-1) \pi}{a}+\mathcal{O}\left(n^{-1}\right)$ as $n \rightarrow \infty$.

Proof. Define $H_{1}(x)=\tan a x$ and $H_{2}(x)=x /\left(b x^{2}-c\right)$. If $(2 n+1) \pi / 2 a=\sqrt{c} / \sqrt{b}$ for some $n \geq 0$ then $\pm \sqrt{c} / \sqrt{b}$ are zeros of $H$. The other zeros of $H$ are precisely the abscissas of the points of intersection of the graphs of $H_{1}$ and $H_{2}$. If $(2 n+1) \pi / 2 a \neq \sqrt{c} / \sqrt{b}$ for all $n \geq 0$ then the zeros of $H$ are just the abscissas of the intersection of $H_{1}$ and $H_{2}$. By looking at the graphs of $H_{1}$ and $H_{2}$ it can be seen that for large indices $n$, we have $\mu_{n}=(n-1) \pi / a+e_{n}$ where $e_{n} \rightarrow 0$. Multiplying by $a$ and taking the sine of both sides yields

$$
\sin a e_{n}=(-1)^{n-1} \frac{\mu_{n} \cos a \mu_{n}}{b \mu_{n}^{2}-c}=(-1)^{n-1} \frac{((n-1) \pi / a+\mathcal{O}(1)) \cos a \mu_{n}}{b((n-1) \pi / a+\mathcal{O}(1))^{2}-c}, n \rightarrow \infty .
$$

Taking the inverse sine and noting that $\sin ^{-1} x=\mathcal{O}(x)$ as $x \rightarrow 0$ we obtain that $e_{n}=\mathcal{O}\left(n^{-1}\right)$.

Theorem 3.2. Let $\left(\mu_{n}\right)_{n \in \mathbb{Z}}$, listed in strictly increasing order, be the real solutions of the equation,

$$
\frac{2 A_{T}}{\gamma A_{e}} \mu \cos \frac{\mu \ell}{\sqrt{\alpha A_{e}}}-\frac{\sqrt{A_{e}}}{\sqrt{\alpha}}\left(\frac{A_{T}^{2}}{\gamma^{2} A_{\mathrm{e}}^{2}} \mu^{2}-\frac{\alpha}{A_{e}}\right) \sin \frac{\mu \ell}{\sqrt{\alpha A_{e}}}=0
$$

where $\mu_{0}=0$ and $\mu_{-n}=-\mu_{n}$. Then the spectrum of $\mathcal{A}$ is given by $\sigma(\mathcal{A})=\left(\lambda_{n}\right)_{n \in \mathbb{Z}}$, where

$$
\lambda_{n}=-\frac{\beta}{2}+\frac{1}{2} \operatorname{sgn}(n) \sqrt{\beta^{2}-4 \mu_{n}^{2}}, \quad n \in \mathbb{Z}^{*}:=\mathbb{Z} \backslash\{0\},
$$

and $\lambda_{0}=0$, and the eigenvalues $\lambda_{n}$ satisfy the asymptotic growth

$$
\lambda_{n}=-\frac{\beta}{2}+\left(\frac{\sqrt{\alpha A_{e}}(n-1) \pi}{\ell}+\mathcal{O}\left(n^{-1}\right)\right) i, \quad n \rightarrow \infty .
$$

In particular, $\sigma(\mathcal{A})=\sigma\left(\mathcal{A}^{*}\right)$. An eigenvector $z_{n}$ of $\mathcal{A}$ associated with the eigenvalue $\lambda_{n}$ is given by

$$
z_{n}=\left(\begin{array}{c}
\varphi_{n} \\
\psi_{n} \\
\eta_{0 n} \\
\eta_{n}
\end{array}\right)=\left(\begin{array}{c}
\cos \frac{\mu_{n} x}{\sqrt{\alpha A_{e}}}-\frac{A_{T} \mu_{n}}{\gamma \sqrt{\alpha A_{e}}} \sin \frac{\mu_{n} x}{\sqrt{\alpha A_{e}}} \\
-\left(\frac{A_{T} \lambda_{n}}{\gamma A_{e}} \cos \frac{\mu_{n} x}{\sqrt{\alpha A_{e}}}+\frac{\sqrt{\alpha} \lambda_{n}}{\sqrt{A_{e}} \mu_{n}} \sin \frac{\mu_{n} x}{\sqrt{\alpha A_{e}}}\right) \\
\frac{1}{\gamma} \\
\frac{1}{\gamma}\left(\cos \frac{\mu_{n} \ell}{\sqrt{\alpha A_{e}}}-\frac{A_{T} \mu_{n}}{\gamma \sqrt{\alpha A_{e}}} \sin \frac{\mu_{n} \ell}{\sqrt{\alpha A_{e}}}\right)
\end{array}\right), \quad n \in \mathbb{Z}
$$

Similarly, an eigenvector $z_{n}^{*}$ of $\mathcal{A}^{*}$ associated to the eigenvalue $\lambda_{n}$ is given by $z_{n}^{*}=\left(\varphi_{n},-\psi_{n}, \eta_{0 n}, \eta_{n}\right)$ for every $n \in \mathbb{Z}$. 
Proof. Note that $\lambda \in \sigma(\mathcal{A})$ if and only if there exists $\left(A, u, h_{0}, h\right) \in \mathcal{D}(\mathcal{A}) \backslash\{0\}$ satisfying the boundary value problem

$$
\left\{\begin{array}{l}
\frac{\partial}{\partial x}\left(\begin{array}{l}
A \\
u
\end{array}\right)=\left(\begin{array}{cc}
0 & -\frac{\lambda+\beta}{\alpha} \\
-\frac{\lambda}{A_{e}} & 0
\end{array}\right)\left(\begin{array}{l}
A \\
u
\end{array}\right) \\
-\frac{A_{e}}{A_{T}} u(0)=\frac{\lambda}{\gamma} A(0), \quad \frac{A_{e}}{A_{T}} u(\ell)=\frac{\lambda}{\gamma} A(\ell)
\end{array}\right.
$$

Consider the equation $\mathcal{A}\left(A, u, h_{0}, h\right)=0$ where $\left(A, u, h_{0}, h\right) \in \mathcal{D}(\mathcal{A})$. Then we have $u=0$ and $A$ is constant. Since $A(0)=\gamma h_{0}$ and $A(\ell)=\gamma h$ we have $h_{0}=h$ and so $\left(A, u, h_{0}, h\right)=c(\gamma, 0,1,1)$ for some $c \in \mathbb{C}$. Hence $0 \in \sigma(\mathcal{A})$. One can check that $-\beta \in \sigma(\mathcal{A})$ with $\beta>0$ if and only if $\ell=-2 A_{T} / \gamma$, hence we exclude this case under the physically relevant assumption $\ell>0$.

Suppose that $\lambda \neq 0$ and $\lambda \neq-\beta$. By diagonalizing the $2 \times 2$ matrix in (3.5) we can obtain that the solution of the ODE is given by

$$
\begin{aligned}
& A(x)=c_{1} \cosh \frac{\sqrt{\lambda(\lambda+\beta)} x}{\sqrt{\alpha A_{e}}}-c_{2} \frac{\sqrt{A_{e}}}{\sqrt{\alpha}} \frac{\sqrt{\lambda(\lambda+\beta)}}{\lambda} \sinh \frac{\sqrt{\lambda(\lambda+\beta)} x}{\sqrt{\alpha A_{e}}} \\
& u(x)=-c_{1} \frac{\sqrt{\alpha}}{\sqrt{A_{e}}} \frac{\lambda}{\sqrt{\lambda(\lambda+\beta)}} \sinh \frac{\sqrt{\lambda(\lambda+\beta)} x}{\sqrt{\alpha A_{e}}}+c_{2} \cosh \frac{\sqrt{\lambda(\lambda+\beta)} x}{\sqrt{\alpha A_{e}}}
\end{aligned}
$$

for some $\left(c_{1}, c_{2}\right) \in \mathbb{C}^{2}$, where the square root denotes any fixed branch of the complex square root; for definiteness we choose the principal branch where the nonpositive real axis is the chosen branch cut.

This and the boundary conditions yield the following homogeneous system of equations

$$
\begin{gathered}
-\left(\frac{A_{T} \lambda}{A_{e} \gamma} \cosh \frac{\sqrt{\lambda(\lambda+\beta)} \ell}{\sqrt{\alpha A_{e}}}+\frac{\sqrt{\alpha}}{\sqrt{A_{e}}} \frac{\lambda}{\sqrt{\lambda(\lambda+\beta)}} \sinh \frac{\sqrt{\lambda(\lambda+\beta)} \ell}{\sqrt{\alpha A_{e}}}\right) c_{1} \\
+\left(\cosh \frac{\sqrt{\lambda(\lambda+\beta)} \ell}{\sqrt{\alpha A_{e}}}+\frac{A_{T}}{A_{e} \gamma} \frac{\sqrt{A_{e}}}{\sqrt{\alpha}} \sqrt{\lambda(\lambda+\beta)} \sinh \frac{\sqrt{\lambda(\lambda+\beta)} \ell}{\sqrt{\alpha A_{e}}}\right) c_{2}=0 \\
\frac{A_{T} \lambda}{A_{e} \gamma} c_{1}+c_{2}=0
\end{gathered}
$$

The above system in the unknowns $c_{1}$ and $c_{2}$ has a nontrivial solution if and only if the determinant of the corresponding matrix is zero and this is equivalent to the equation

$$
F(w):=\frac{2 A_{T}}{A_{e} \gamma} w \cosh \frac{w \ell}{\sqrt{\alpha A_{e}}}+\frac{\sqrt{A_{e}}}{\sqrt{\alpha}}\left(\frac{A_{T}^{2}}{A_{\mathrm{e}}^{2} \gamma^{2}} w^{2}+\frac{\alpha}{A_{e}}\right) \sinh \frac{w \ell}{\sqrt{\alpha A_{e}}}=0
$$

where we put $w=\sqrt{\lambda(\lambda+\beta)}$.

Let us consider the special case where $\beta=0$. In this case, $\lambda \in \sigma(\mathcal{A})$ if and only if $F(\lambda)=0$. However, since $\mathcal{A}$ is skew-adjoint, its spectrum must lie on the imaginary axis. This implies that all zeros of $F$ are purely imaginary. Letting $\lambda=i \mu$, where $\mu \in \mathbb{R}$, we can see that $F(i \mu)=0$ is equivalent to the equation (3.1). Using this for the case $\beta \geq 0$, we can see from (3.8) that $\lambda \in \sigma(\mathcal{A})$ if and only if $\sqrt{\lambda(\lambda+\beta)}=i \mu$ for some $\mu \in \mathbb{R}$ that satisfies (3.1). The asymptotic behavior (3.3) of the eigenvalues follows from the asymptotic behavior of the solutions of (3.1) given by the previous lemma

$$
\mu_{n}=\frac{\sqrt{\alpha A_{e}}(n-1) \pi}{\ell}+\mathcal{O}\left(n^{-1}\right), \quad n \rightarrow \infty .
$$

The fact that the spectra of $\mathcal{A}$ and $\mathcal{A}^{*}$ coincide comes from the symmetry of the spectrum of $\mathcal{A}$ with respect to the real axis. 
Choosing $c_{1}=1$ and $c_{2}=-\frac{A_{T} \lambda_{n}}{\gamma A_{e}}$ in (3.6) and (3.7) gives the first and second components of the eigenvector $z_{n}$. The third and fourth components are due to the boundary conditions $\eta_{0 n}=\frac{1}{\gamma} \varphi_{n}(0)$ and $\eta_{n}=\frac{1}{\gamma} \varphi_{n}(\ell)$. Finally, since $z_{n}$ is an eigenvector of $\mathcal{A}$ corresponding to $\lambda_{n}$ we have

$$
\left(\lambda_{n} I-\mathcal{A}^{*}\right) z_{n}^{*}=\left(\begin{array}{c}
\lambda_{n} \varphi_{n}+A_{e}\left(\psi_{n}\right)_{x} \\
-\lambda_{n} \psi_{n}-\alpha\left(\varphi_{n}\right)_{x}-\beta \psi_{n} \\
\lambda_{n} \eta_{0 n}+\frac{A_{e}}{A_{T}} \psi_{n}(0) \\
\lambda_{n} \eta_{n}-\frac{A_{e}}{A_{T}} \psi_{n}(\ell)
\end{array}\right)=\left(\begin{array}{l}
0 \\
0 \\
0 \\
0
\end{array}\right),
$$

and so $z_{n}^{*}$ is an eigenvector of $\mathcal{A}^{*}$ corresponding to the eigenvalue $\lambda_{n}$.

If $\beta>2 \mu_{n}$, then the eigenvalue $\lambda_{n}$ is real and negative. This implies that $z(t, x)=\left(\mathrm{e}^{\mathcal{A} t}\right) z_{n}(x)=\mathrm{e}^{\lambda_{n} t} z_{n}(x)$ monotonically decays to zero as time goes to infinity. This means that when the fluid inside the tube and tanks is sufficiently viscous, then there are solutions which decay to the equilibrium state without oscillations.

With or without viscosity, we have seen in Theorem 3.2 that $0 \in \sigma(\mathcal{A})$ and this means that the system is not stable in $\mathcal{X}$. The eigenspace associated with the eigenvalue 0 is the one-dimensional subspace $\mathcal{X}_{0}:=$ $\{c(\gamma, 0,1,1): c \in \mathbb{C}\}$. Moreover, $\mathcal{N}\left(\mathcal{A}^{*}\right)=\mathcal{X}_{0}$ and so $\mathcal{A}$ and its adjoint have the same kernel. The state $\left(A_{e}, 0, h_{0 e}, h_{e}\right)+c(\gamma, 0,1,1)$ for $c \neq 0$ is also an equilibrium of the nonlinear system but corresponds to different parameters $V, p_{f 0}$ and $p_{f}$.

It is easy to check that the space $\mathcal{X}_{0}$ is invariant under the action of the group $\left(\mathrm{e}^{\mathcal{A} t}\right)_{t \in \mathbb{R}}$ and its adjoint group. If $z \in \mathcal{X}_{0}^{\perp}$ and $w \in \mathcal{X}_{0}$ then $\left\langle\mathrm{e}^{\mathcal{A} t} z, w\right\rangle_{\mathcal{X}}=\left\langle z, \mathrm{e}^{\mathcal{A}^{*} t} w\right\rangle_{\mathcal{X}}=0$ by the invariance of $\mathcal{X}_{0}$ under $\left(\mathrm{e}^{\mathcal{A}^{*} t}\right)_{t \in \mathbb{R}}$. Hence $\mathcal{X}_{0}^{\perp}$ is invariant under $\left(\mathrm{e}^{\mathcal{A} t}\right)_{t \in \mathbb{R}}$, i.e., $\mathrm{e}^{\mathcal{A} t} \mathcal{X}_{0}^{\perp} \subset \mathcal{X}_{0}^{\perp}$ for all $t \in \mathbb{R}$. From ([22], Prop. 2.4.3), the restricted group $\left(\left.\mathrm{e}^{\mathcal{A} t}\right|_{\mathcal{X}_{0}^{\perp}}\right)_{t \in \mathbb{R}}$ is a $\mathcal{C}_{0}$-group on $\mathcal{X}_{0}^{\perp}$ whose generator is given by the part of $\mathcal{A}$ in $\mathcal{X}_{0}^{\perp}$, denoted by $\mathcal{A}_{p}$. A similar argument shows that $\left.\mathrm{e}^{\mathcal{A}^{*} t}\right|_{\mathcal{X}_{0}^{\perp}}=\mathrm{e}^{\mathcal{A}_{p}^{*} t}$ for all $t \in \mathbb{R}$ where $\mathcal{A}_{p}^{*}$ is the part of $\mathcal{A}^{*}$ in $\mathcal{X}_{0}^{\perp}$. It is easily seen that $\mathcal{A}_{p}^{*}=\left(\mathcal{A}_{p}\right)^{*}$, which means that taking the part in a closed invariant subspace and taking the adjoint commute. Since $\mathcal{A}_{p}$ and $\mathcal{A}_{p}^{*}$ also have compact resolvents we have $\sigma\left(\mathcal{A}_{p}\right)=\sigma\left(\mathcal{A}_{p}^{*}\right)=\sigma(\mathcal{A}) \backslash\{0\}, \rho\left(\mathcal{A}_{p}\right)=\rho\left(\mathcal{A}_{p}^{*}\right)=\rho(\mathcal{A}) \cup\{0\}$. The semigroup generated by $\mathcal{A}_{p}$ will be used in the next section.

Let us characterize the nonzero eigenvalues of $\mathcal{A}$ as the viscosity $\beta$ increases. In the following discussions the equality (3.2) is used. If $\beta \in\left[0,2 \mu_{1}\right)$ then all the nonzero eigenvalues have nonzero imaginary parts and $\left|\lambda_{n}\right|=\left|\mu_{n}\right|$ for all $n \in \mathbb{Z}$. Therefore as $\beta$ increases on this interval, the eigenvalues are rotated positively (that is, counterclockwise) around the origin and all the eigenvalues lie on the line $\Re z=-\beta / 2$. If $\beta=2 \mu_{1}$ then the two eigenvalues $\lambda_{ \pm 1}$ coincide and both are equal to $-\beta / 2$. Suppose that $\beta \in\left[2 \mu_{1}, 2 \mu_{2}\right)$. As $\beta$ increases in this interval, $\lambda_{n}$ for $|n|>2$ is again rotated in the same manner as before. However, the eigenvalue $\lambda_{1}$ now goes to the right along the real axis while $\lambda_{-1}$ goes to the left along the real axis, faster than $\Re \lambda_{ \pm 2}$. When $\beta=2 \mu_{2}$, the eigenvalues $\lambda_{ \pm 2}$ coincide while $\lambda_{-1}$ is on the left of $\lambda_{ \pm 2}$. The same behavior holds for the other intervals $\left[2 \mu_{n}, 2 \mu_{n+1}\right), n \geq 2$. Thus the larger $\beta$, the more eigenvalues there are on the interval $(-\beta / 2,0)$ and there are eigenfunctions which decay slower than those for smaller $\beta$. The eigenvalues that move to the right approach 0 as $\beta$ increases.

If we denote by $\zeta_{n}$ the eigenvector of $\mathcal{A}$ corresponding to $\lambda_{n}=i \mu_{n}$ when $\beta=0$ and by $z_{n}$ the eigenvector of $\mathcal{A}$ when $\beta>0$ then for all $n$ such that $\beta<2\left|\mu_{n}\right|$ we have $\left|\lambda_{n}\right|=\left|\mu_{n}\right|$,

$$
\left\|z_{n}\right\|_{\mathcal{X}}=\left\|z_{n}^{*}\right\|_{\mathcal{X}}=\left\|\zeta_{n}\right\|_{\mathcal{X}}=\left\|\zeta_{-n}\right\|_{\mathcal{X}}
$$

and a straightforward calculation gives

$$
\left\|\zeta_{n}\right\|_{\mathcal{X}}^{2}=\frac{A_{T}^{2} \ell \mu_{n}^{2}}{\gamma^{2} \alpha A_{\mathrm{e}}^{2}}+\frac{\ell}{A_{e}}+\frac{A_{T}}{\gamma A_{e}}+\frac{A_{T}}{\gamma A_{e}}\left(\cos \frac{\mu_{n} \ell}{\sqrt{\alpha A_{e}}}-\frac{A_{T} \mu_{n}}{\gamma \sqrt{\alpha A_{e}}} \sin \frac{\mu_{n} \ell}{\sqrt{\alpha A_{e}}}\right)^{2} .
$$

Theorem 3.3. Let $\beta \geq 0$. Then we have the following:

1. If $\beta \neq 2 \mu_{n}$ for all $n \in \mathbb{N}$ then the normalized eigenvectors $\left(z_{n} /\left\|z_{n}\right\|_{\mathcal{X}}\right)_{n \in \mathbb{Z}}$ of $\mathcal{A}$ form a Riesz basis for $\mathcal{X}$. If $\beta=0$ then this Riesz basis is in fact an orthonormal basis. 
2. If $\beta=2 \mu_{n}$ for some $n \in \mathbb{N}$ then the sequence $\left(z_{n} /\left\|z_{n}\right\|_{\mathcal{X}}\right)_{n \in \mathbb{Z} \backslash\{-n\}} \cup\left\{Z /\|Z\|_{\mathcal{X}}\right\}$, where

$$
Z=\left(0,-\lambda_{n}^{-1} \psi_{n}, 0,0\right)^{\top}
$$

is a generalized eigenvector of $\mathcal{A}$ corresponding to $\lambda_{n}$ satisfying $\left(\lambda_{n} I-\mathcal{A}\right) Z=z_{n}$, forms a Riesz basis for $\mathcal{X}$.

Similar results for the generator $\mathcal{A}^{*}$ hold, however, in 2 the vector $Z$ should be replaced by the generalized eigenvector $Z^{*}:=-Z$ of $\mathcal{A}^{*}$ corresponding to $\lambda_{n}$.

Proof. First consider the case where $\beta=0$. Applying ([1], Prop. III.6.1) to the operator $\mathcal{A}_{p}$, the normalized eigenvectors $\left(z_{n} /\left\|z_{n}\right\|_{\mathcal{X}}\right)_{n \in \mathbb{Z}^{*}}$ forms an orthonormal basis for $\mathcal{X}_{0}^{\perp}$. Therefore combined with the normalized eigenvector associated with the eigenvalue 0 , the sequence $\left(z_{n} /\left\|z_{n}\right\|_{\mathcal{X}}\right)_{n \in \mathbb{Z}}$ form an orthonormal basis for $\mathcal{X}=$ $\mathcal{X}_{0} \oplus \mathcal{X}_{0}^{\perp}$. Now suppose that $\beta>0$ and $\beta \neq 2 \mu_{n}$ for all $n \in \mathbb{N}$. Again, let $\zeta_{n}$ be the eigenvector of $\mathcal{A}$ corresponding to the eigenvalue $\lambda_{n}=i \mu_{n}$ for the case where there is no viscosity, i.e., $\beta=0$. The first part of 1 follows from Theorem 2.4 of [25], once we have shown that $\left(z_{n} /\left\|z_{n}\right\|_{\mathcal{X}}\right)_{n \in \mathbb{Z}}$ and $\left(\zeta_{n} /\left\|\zeta_{n}\right\|_{\mathcal{X}}\right)_{n \in \mathbb{Z}}$ are quadratically close in the sense that

$$
\sum_{n \in \mathbb{Z}}\left\|\frac{z_{n}}{\left\|z_{n}\right\|_{\mathcal{X}}}-\frac{\zeta_{n}}{\left\|\zeta_{n}\right\|_{\mathcal{X}}}\right\|_{\mathcal{X}}^{2}<\infty .
$$

Let $N$ be the largest integer such that $\beta>2 \mu_{N}$. From (3.4) and (3.10)

$$
\frac{z_{n}}{\left\|z_{n}\right\|_{\mathcal{X}}}-\frac{\zeta_{n}}{\left\|\zeta_{n}\right\|_{\mathcal{X}}}=\left(0, \frac{\lambda_{n}-i \mu_{n}}{i \mu_{n}} \frac{\zeta_{n 2}}{\left\|\zeta_{n}\right\|_{\mathcal{X}}}, 0,0\right), \quad|n|>N
$$

where $\zeta_{n 2}$ is the second component of $\zeta_{n}$. It can be seen from (3.2) that $\left|\lambda_{n}-i \mu_{n}\right| \rightarrow \beta / 2$ as $|n| \rightarrow \infty$ and in particular the sequence $\left(\lambda_{n}-i \mu_{n}\right)_{n \in \mathbb{Z}}$ is bounded. Because $\left\|\zeta_{n 2}\right\|_{L^{2}(0, \ell)} \leq \sqrt{\alpha}\left\|\zeta_{n}\right\|_{\mathcal{X}}$ it follows from (3.13) that

$$
\sum_{|n|>N}\left\|\frac{z_{n}}{\left\|z_{n}\right\|_{\mathcal{X}}}-\frac{\zeta_{n}}{\left\|\zeta_{n}\right\|_{\mathcal{X}}}\right\|_{\mathcal{X}}^{2} \leq \sum_{|n|>N} \frac{C}{\mu_{n}^{2}}
$$

for some constant $C>0$. The last sum is finite because of (3.9). As a consequence, (3.12) is satisfied.

Finally, consider the case where $\beta=2 \mu_{n}$ for some $n \in \mathbb{N}$. Let us verify that $Z$ satisfies $\left(\lambda_{n} I-\mathcal{A}\right) Z=z_{n}$. Indeed,

$$
\left(\lambda_{n} I-\mathcal{A}\right) Z-z_{n}=\left(\begin{array}{c}
A_{e}\left(-\lambda_{n}^{-1} \psi_{n}\right)_{x}-\varphi_{n} \\
\lambda_{n}\left(-\lambda_{n}^{-1} \psi\right)-\beta \lambda_{n}^{-1} \psi_{n}-\psi_{n} \\
\frac{A_{e}}{A_{T}}\left(-\lambda_{n}^{-1} \psi_{n}\right)(0)-\eta_{0} \\
-\frac{A_{e}}{A_{T}}\left(-\lambda_{n}^{-1} \psi_{n}\right)(\ell)-\eta_{n}
\end{array}\right)=\left(\begin{array}{c}
-\lambda_{n}^{-1}\left(\lambda_{n} \varphi_{n}+A_{e}\left(\psi_{n}\right)_{x}\right) \\
-\psi_{n}+2 \psi_{n}-\psi_{n} \\
-\lambda_{n}^{-1}\left(\lambda_{n} \eta_{0 n}+\frac{A_{e}}{A_{T}} \psi_{n}(0)\right) \\
-\lambda_{n}^{-1}\left(\lambda_{n} \eta_{n}-\frac{A_{e}}{A_{T}} \psi_{n}(\ell)\right)
\end{array}\right)
$$

and this is zero because $z_{n}$ is an eigenvector of $\mathcal{A}$ corresponding to $\lambda_{n}=-\beta / 2$. The same argument as in the previous case shows that the sequences $\left(z_{n} /\left\|z_{n}\right\|_{\mathcal{X}}\right)_{n \in \mathbb{Z} \backslash\{-n\}} \cup\left\{Z /\|Z\|_{\mathcal{X}}\right\}$ and $\left(\zeta_{n} /\left\|\zeta_{n}\right\|_{\mathcal{X}}\right)_{n \in \mathbb{Z}}$ are quadratically close and hence part 2 also follows from the Riesz basis generation result in Theorem 2.4 of [25].

Let $\left(\tilde{z}_{n}\right)_{n \in \mathbb{Z}}$ be the sequence biorthogonal to the Riesz basis $\left(z_{n}^{*} /\left\|z_{n}^{*}\right\|_{\mathcal{X}}\right)_{n \in \mathbb{Z}}$ if $\beta \neq 2 \mu_{n}$ for all $n \in \mathbb{N}$ or to the Riesz basis $\left(z_{n}^{*} /\left\|z_{n}^{*}\right\|_{\mathcal{X}}\right)_{n \in \mathbb{Z} \backslash\{-n\}} \cup\left\{Z^{*} /\left\|Z^{*}\right\|_{\mathcal{X}}\right\}$ if $\beta=2 \mu_{n}$ for some $n \in \mathbb{N}$. The result we have just proved implies that every $z \in \mathcal{X}$ can be expressed uniquely as a Fourier series

$$
z=\sum_{n \in \mathbb{Z}}\left\langle z, \tilde{z}_{n}\right\rangle \mathcal{X} \frac{z_{n}^{*}}{\left\|z_{n}^{*}\right\|_{\mathcal{X}}}
$$


whenever $\beta \neq 2 \mu_{n}$ for all $n \in \mathbb{N}$ and a similar equation holds for the other case. For all square-summable sequences $\left(a_{n}\right)_{n \in \mathbb{Z}}$ we have

$$
c\left(\sum_{n \in \mathbb{Z}}\left|a_{n}\right|^{2}\right)^{1 / 2} \leq\left\|\sum_{n \in \mathbb{Z}} \frac{a_{n} z_{n}^{*}}{\left\|z_{n}^{*}\right\|_{\mathcal{X}}}\right\|_{\mathcal{X}} \leq C\left(\sum_{n \in \mathbb{Z}}\left|a_{n}\right|^{2}\right)^{1 / 2}
$$

for some $c, C>0$ independent of $\left(a_{n}\right)_{n \in \mathbb{Z}}$. Furthermore, the sequence $\left(\left\langle z, \tilde{z}_{n}\right\rangle_{\mathcal{X}}\right)_{n \in \mathbb{Z}^{*}}$ is square-summable for each $z \in \mathcal{X}$. Since $\mathrm{e}^{\mathcal{A}^{*} t} z_{n}^{*}=\mathrm{e}^{\lambda_{n} t} z_{n}^{*}$ for all $n \in \mathbb{Z}$ it follows from (3.14) and the continuity of $\mathrm{e}^{\mathcal{A}^{*} t}$ that when $\beta \neq 2 \mu_{n}$ for all $n \in \mathbb{N}$ the group generated by $\mathcal{A}^{*}$ can be written as

$$
\mathrm{e}^{\mathcal{A}^{*} t} z=\sum_{n \in \mathbb{Z}^{*}} \mathrm{e}^{\lambda_{n} t}\left\langle z, \tilde{z}_{n}\right\rangle \mathcal{X} \frac{z_{n}^{*}}{\left\|z_{n}^{*}\right\|_{\mathcal{X}}}
$$

for every $z \in \mathcal{X}$ and $t \in \mathbb{R}$. If $\beta=2 \mu_{n}$ for some $n \in \mathbb{N}$ then the group is given by

$$
\mathrm{e}^{\mathcal{A}^{*} t} z=\left\langle z, \tilde{z}_{-n}\right\rangle\left(\mathrm{e}^{\lambda_{n} t} Z^{*}-t \mathrm{e}^{\lambda_{n} t} z_{n}^{*}\right)+\sum_{n \in \mathbb{Z} \backslash\{-n\}} \mathrm{e}^{\lambda_{n} t}\left\langle z, \tilde{z}_{n}\right\rangle \mathcal{X} \frac{z_{n}^{*}}{\left\|z_{n}^{*}\right\|_{\mathcal{X}}}
$$

for every $z \in \mathcal{X}$ and $t \in \mathbb{R}$. Similar characterizations for the group generated by $\mathcal{A}$ hold. The reason why we choose to expand the adjoint semigroup is that we will use a duality argument in the proof of Theorem 4.3. Now we have the following stability result.

Theorem 3.4. Let $\beta>0$. The $\mathcal{C}_{0}$-semigroups $\left(\mathrm{e}^{\mathcal{A}_{p} t}\right)_{t \geq 0}$ and $\left(\mathrm{e}^{\mathcal{A}_{p}^{*} t}\right)_{t \geq 0}$ generated by $\mathcal{A}_{p}$ and $\mathcal{A}_{p}^{*}$ are uniformly exponentially stable, i.e., there exist constants $M \geq 1$ and $\omega>0$ such that

$$
\left\|\mathrm{e}^{\mathcal{A}_{p} t}\right\|_{\mathcal{L}\left(\mathcal{X}_{0}^{\perp}\right)}=\left\|\mathrm{e}^{\mathcal{A}_{p}^{*} t}\right\|_{\mathcal{L}\left(\mathcal{X}_{0}^{\perp}\right)} \leq M \mathrm{e}^{-\omega t}, \quad t \geq 0 .
$$

Furthermore, $\omega\left(\mathcal{A}_{p}\right)=s\left(\mathcal{A}_{p}\right)$ where $s\left(\mathcal{A}_{p}\right)$ is the spectral bound of $\mathcal{A}_{p}$ and $\omega\left(\mathcal{A}_{p}\right)$ is the growth bound of the semigroup generated by $\mathcal{A}_{p}$. For every $z_{0} \in \mathcal{X}$, if $z=\mathrm{e}^{\mathcal{A} t} z_{0} \in C([0, \infty), \mathcal{X})$ is the mild solution of $(\mathrm{ACP})$ corresponding to the initial data $z_{0}$ then $\left\|z(t)-P z_{0}\right\|_{\mathcal{X}} \leq M\left\|z_{0}\right\|_{\mathcal{X}} \mathrm{e}^{-\omega t}$ for all $t \geq 0$, where $P$ is the orthogonal projection of $\mathcal{X}$ onto $\mathcal{X}_{0}$.

Proof. The first and second parts follow immediately from (3.16) and (3.17). For the last part, let $Q$ be the orthogonal projection of $\mathcal{X}$ onto $\mathcal{X}_{0}^{\perp}$ so that every $z_{0} \in \mathcal{X}$ can be written uniquely as $z_{0}=P z_{0}+Q z_{0}$. Since the restriction of $\mathrm{e}^{\mathcal{A} t}$ to $\mathcal{X}_{0}$ is just the identity operator on $\mathcal{X}_{0}$ we have $z(t)=P z_{0}+\mathrm{e}^{\mathcal{A}_{p} t} Q z_{0}$, and the required estimate follows from (3.18) and the fact that $\|Q\| \leq 1$.

The eigenvalue 0 is removed by restricting the state space to the orthogonal complement of the eigenspace corresponding to the eigenvalue zero. Define the volume functional $\mathcal{V}: \mathcal{X} \rightarrow \mathbb{C}$ by

$$
\mathcal{V}\left(A, u, h_{0}, h\right)=\int_{0}^{\ell} A(x) \mathrm{d} x+A_{T} h_{0}+A_{T} h .
$$

It is clear that $\mathcal{V}$ is a bounded linear functional on $\mathcal{X}$. Recall that $z=z_{e}+\tilde{z}$ where $z=\left(A, u, h_{0}, h\right), z_{e}=$ $\left(A_{e}, 0, h_{0 e}, h_{e}\right)$ and $\tilde{z}=\left(\tilde{A}, \tilde{u}, \tilde{h}_{0}, \tilde{h}\right)$ are the state, the equilibrium state and the deviation of the state from the equilibrium, respectively. By the conservation of mass we must have $\mathcal{V}(z)=\mathcal{V}\left(z_{e}\right)=V$ and this is equivalent to $\mathcal{V}(\tilde{z})=0$, i.e., $\tilde{z} \in \mathcal{N}(\mathcal{V})$. One can check that $\mathcal{N}(\mathcal{V})=\mathcal{X}_{0}^{\perp}$. This means that $\mathcal{X}_{0}^{\perp}$ is the natural state space for the deviations. Also, if $z(t, x)=\mathrm{e}^{\mathcal{A}_{p} t} z^{0}(x)$ is the solution of the system then $\mathcal{V}(z(t, \cdot))=0$ for every $t \geq 0$ whenever $z^{0} \in \mathcal{X}_{0}^{\perp}$. For this reason, we consider $\mathcal{X}_{0}^{\perp}$ to be the state space in the next section. 


\section{A BOUndary CONTROL SYSTEM}

Consider time varying control pressures $p_{f 0}(t)$ and $p_{f}(t)$ applied to the left and the right tank, respectively. Linearizing about the numbers $p_{f 0}^{*}$ and $p_{f}^{*}$ we have

$$
\begin{aligned}
& \tilde{A}(t, 0)=\gamma \tilde{h}_{0}(t)+\frac{\gamma}{\rho g} \tilde{p}_{f 0}(t), \\
& \tilde{A}(t, \ell)=\gamma \tilde{h}(t)+\frac{\gamma}{\rho g} \tilde{p}_{f}(t)
\end{aligned}
$$

where $\tilde{p}_{f 0}(t)=p_{f 0}(t)-p_{f 0}^{*}$ and $\tilde{p}_{f}(t)=p_{f}(t)-p_{f}^{*}$. Again for simplicity, we ignore the tildas and we let $p_{0}=\frac{\gamma}{\rho g} p_{f 0}$ and $p_{1}=\frac{\gamma}{\rho g} p_{f}$. In this scenario, we have the system (1.8) with the boundary conditions

$$
A(t, 0)=\gamma h_{0}(t)+p_{0}(t), \quad A(t, \ell)=\gamma h(t)+p_{1}(t), \quad t>0 .
$$

Definition 4.1. For $A^{0}, u^{0} \in L^{2}(0, \ell), h_{0}^{0}, h^{0} \in \mathbb{C}$ and $p_{0}, p_{1} \in L_{\text {loc }}^{2}([0, \infty), \mathbb{C})$, the tuple $\left(A, u, h_{0}, h\right)$ such that $A, u \in C\left([0, \infty), L^{2}(0, \ell)\right)$ and $h_{0}, h \in C([0, \infty), \mathbb{C})$ is called a weak solution of the system (1.8) with initial conditions (1.10) and boundary conditions (4.1) if

$$
\left\{\begin{aligned}
\langle A(t), \varphi\rangle_{L^{2}}+ & \gamma A_{T}\left(h_{0}(t), \eta_{0}\right)+\gamma A_{T}(h(t), \eta) \\
= & \left\langle A^{0}, \varphi\right\rangle_{L^{2}}+\gamma A_{T}\left(h_{0}^{0}, \eta_{0}\right)+\gamma A_{T}\left(h^{0}, \eta\right)+A_{e} \int_{0}^{t}\left\langle u(\sigma), \varphi_{x}\right\rangle_{L^{2}} \mathrm{~d} \sigma \\
\langle u(t), \psi\rangle_{L^{2}}= & \left\langle u^{0}, \psi\right\rangle_{L^{2}}+\alpha \int_{0}^{t}\left\langle A(\sigma), \psi_{x}\right\rangle_{L^{2}} \mathrm{~d} \sigma-\beta \int_{0}^{t}\langle u(\sigma), \psi\rangle_{L^{2}} \mathrm{~d} \sigma \\
& +\alpha \int_{0}^{t}\left(\gamma h_{0}(\sigma)+p_{0}(\sigma), \psi(0)\right)-\left(\gamma h(\sigma)+p_{1}(\sigma), \psi(\ell)\right) \mathrm{d} \sigma
\end{aligned}\right.
$$

for every $t \geq 0, \varphi, \psi \in H^{1}(0, \ell)$ and $\eta_{0}, \eta \in \mathbb{C}$ such that $\varphi(0)=\gamma \eta_{0}$ and $\varphi(\ell)=\gamma \eta$.

To prove the existence of such weak solutions, the system will be expressed as a boundary control system using well-known results in control theory. Because the velocity component of the eigenvector corresponding to the eigenvalue 0 vanishes, the system is not approximately controllable in $\mathcal{X}$, cf. the observation operator $\mathcal{B}^{*}$ in Theorem 4.2 below. For this reason the system is restricted to the state space $\mathcal{X}_{0}^{\perp}$.

Denote by $\mathcal{Z}=\left(H^{1}(0, \ell) \times H^{1}(0, \ell) \times \mathbb{C}^{2}\right) \cap \mathcal{X}_{0}^{\perp}$ the solution space endowed with the product norm of $H^{1}(0, \ell) \times H^{1}(0, \ell) \times \mathbb{C}^{2}$. Our input space is $\mathbb{C}^{2}$ and the state space is $\mathcal{X}_{0}^{\perp}$. Note that $\mathcal{Z}$ is continuously embedded in $\mathcal{X}_{0}^{\perp}$. Let $\mathcal{D}\left(\mathcal{A}_{p}^{*}\right)$ be endowed with the graph norm. Then $\mathcal{D}\left(\mathcal{A}_{p}^{*}\right) \subset \mathcal{X}_{0}^{\perp} \subset \mathcal{D}\left(\mathcal{A}_{p}^{*}\right)^{\prime}$ with continuous and dense embeddings and we have

$$
\langle z, \zeta\rangle_{\mathcal{D}\left(\mathcal{A}_{p}^{*}\right)^{\prime} \times \mathcal{D}\left(\mathcal{A}_{p}^{*}\right)}=\langle z, \zeta\rangle_{\mathcal{X}}, \quad \forall z \in \mathcal{X}_{0}^{\perp}, \zeta \in \mathcal{D}\left(\mathcal{A}_{p}^{*}\right)
$$

Furthermore, we can see that $\mathcal{A}_{p}^{*} \in \mathcal{L}\left(\mathcal{D}\left(\mathcal{A}_{p}^{*}\right), \mathcal{X}_{0}^{\perp}\right)$ so that $\left(\mathcal{A}_{p}^{*}\right)^{*} \in \mathcal{L}\left(\mathcal{X}_{0}^{\perp}, \mathcal{D}\left(\mathcal{A}_{p}^{*}\right)^{\prime}\right)$, where the state space $\mathcal{X}_{0}^{\perp}$ is identified with its dual. The operator $\left(\mathcal{A}_{p}^{*}\right)^{*}$ can be viewed as an extension of $\mathcal{A}_{p}$ to $\mathcal{X}_{0}^{\perp}$. For more details on the interpolation and extrapolation spaces for semigroups the reader may consult ([4], pp. 123-127).

Let $\mathcal{F}: \mathcal{Z} \rightarrow \mathcal{X}_{0}^{\perp}$ and $\mathcal{G}: \mathcal{Z} \rightarrow \mathbb{C}^{2}$ be given by

$$
\mathcal{F}\left(A, u, h_{0}, h\right)=\left(\begin{array}{c}
-A_{e} u_{x} \\
-\alpha A_{x}-\beta u \\
-\frac{A_{e}}{A_{T}} u(0) \\
\frac{A_{e}}{A_{T}} u(\ell)
\end{array}\right)
$$

and

$$
\mathcal{G}\left(A, u, h_{0}, h\right)=\left(\begin{array}{c}
A(0)-\gamma h_{0} \\
A(\ell)-\gamma h
\end{array}\right)
$$


Note that $\mathcal{F} \in \mathcal{L}\left(\mathcal{Z}, \mathcal{X}_{0}^{\perp}\right), \mathcal{G} \in \mathcal{L}\left(\mathcal{Z}, \mathbb{C}^{2}\right), \mathcal{N}(\mathcal{G})=\mathcal{D}\left(\mathcal{A}_{p}\right), \mathcal{R}(\mathcal{G})=\mathbb{C}^{2}$ and $\left.\mathcal{F}\right|_{\mathcal{D}\left(\mathcal{A}_{p}\right)}=\mathcal{A}_{p}$. As a consequence, $(\mathcal{F}, \mathcal{G})$ is a boundary control system. Then according to Proposition 10.1.2 of [22], there exists a unique operator $\mathcal{B} \in \mathcal{L}\left(\mathbb{C}^{2}, \mathcal{D}\left(\mathcal{A}_{p}^{*}\right)^{\prime}\right)$, called the control operator, such that $\mathcal{F} z=\left(\left(\mathcal{A}_{p}^{*}\right)^{*}+\mathcal{B G}\right) z$ for all $z \in \mathcal{Z}$. A characterization of this control operator is given in the following theorem.

Theorem 4.2. The input control operator $\mathcal{B} \in \mathcal{L}\left(\mathbb{C}^{2}, \mathcal{D}\left(\mathcal{A}_{p}^{*}\right)^{\prime}\right)$ is given by

$$
\mathcal{B}\left(c_{1}, c_{2}\right)=-\left(\mathcal{A}_{p}^{*}\right)^{*}\left(\left(\begin{array}{c}
\kappa \\
0 \\
\frac{1}{\gamma}(\kappa-1) \\
\frac{1}{\gamma} \kappa
\end{array}\right) c_{1}+\left(\begin{array}{c}
\kappa \\
0 \\
\frac{1}{\gamma} \kappa \\
\frac{1}{\gamma}(\kappa-1)
\end{array}\right) c_{2}\right),
$$

where $\left(c_{1}, c_{2}\right) \in \mathbb{C}^{2}$ and $\kappa=\frac{A_{T}}{\gamma \ell+2 A_{T}}$. Its adjoint $\mathcal{B}^{*} \in \mathcal{L}\left(\mathcal{D}\left(\mathcal{A}_{p}^{*}\right), \mathbb{C}^{2}\right)$ is given by

$$
\mathcal{B}^{*}\left(B, v, g_{0}, g\right)=\left(\begin{array}{c}
v(0) \\
-v(\ell)
\end{array}\right), \quad\left(B, v, g_{0}, g\right) \in \mathcal{D}\left(\mathcal{A}_{p}^{*}\right)
$$

Proof. Given $\left(c_{1}, c_{2}\right) \in \mathbb{C}^{2}$, consider the problem $\mathcal{F}\left(A, u, h_{0}, h\right)=0$ with boundary conditions $\mathcal{G}\left(A, u, h_{0}, h\right)=$ $\left(c_{1}, c_{2}\right)$. This implies $u=0, A$ is constant, $A-\gamma h_{0}=c_{1}$ and $A-\gamma h=c_{2}$. Since $\left(A, u, h_{0}, h\right) \in \mathcal{X}_{0}^{\perp}=\mathcal{N}(\mathcal{V})$, $A \ell+A_{T} h_{0}+A_{T} h=0$. Note that the unique solution of $\mathcal{F} z=0$ with $\mathcal{G} z=\left(c_{1}, c_{2}\right)$ is given by $-\left(\mathcal{A}_{p}^{*}\right)^{*} z=\mathcal{B}\left(c_{1}, c_{2}\right)$. Solving the linear system for $\left(A, h_{0}, h\right) \in \mathbb{C}^{3}$ in terms of $c_{1}$ and $c_{2}$ we can see that $\mathcal{B}: \mathbb{C}^{2} \rightarrow \mathcal{D}\left(\mathcal{A}_{p}^{*}\right)^{\prime}$ is given by (4.3).

For $z=\left(A, u, h_{0}, h\right) \in \mathcal{Z}$ and $\zeta=\left(B, v, g_{0}, g\right) \in \mathcal{D}\left(\mathcal{A}_{p}^{*}\right)$ we obtain from (4.2) that

$$
\begin{aligned}
\left\langle\mathcal{G} z, \mathcal{B}^{*} \zeta\right\rangle_{\mathbb{C}^{2}} & =\langle\mathcal{B G} z, \zeta\rangle_{\mathcal{D}\left(\mathcal{A}_{p}^{*}\right)^{\prime} \times \mathcal{D}\left(\mathcal{A}_{p}^{*}\right)}=\left\langle\mathcal{F} z-\left(\mathcal{A}_{p}^{*}\right)^{*} z, \zeta\right\rangle_{\mathcal{D}\left(\mathcal{A}_{p}^{*}\right)^{\prime} \times \mathcal{D}\left(\mathcal{A}_{p}^{*}\right)} \\
& =\langle\mathcal{F} z, \zeta\rangle_{\mathcal{X}}-\left\langle z, \mathcal{A}_{p}^{*} \zeta\right\rangle_{\mathcal{X}} .
\end{aligned}
$$

Integrating by parts and using the surjectivity of $\mathcal{G}$ we obtain (4.4).

In the above theorem, we have a representation of the control operator $\mathcal{B}$ in terms of the extension of the operator $\mathcal{A}_{p}$. However, the more important item to use in the controllability of the boundary control system, at least in our case, is the adjoint $\mathcal{B}^{*}$ of the control operator.

We shall make use of the Riesz basis approach to study the above boundary control system. We refer to [13] for various examples in this direction. From Theorem 3.2, the eigenvalues of $\mathcal{A}_{p}^{*}$ satisfy

$$
\liminf _{|n| \rightarrow \infty}\left|\Im \lambda_{n}-\Im \lambda_{n+1}\right|=\frac{\sqrt{\alpha A_{e}} \pi}{\ell}
$$

and, in particular the uniform gap property

$$
\gamma_{0}:=\inf _{\substack{\lambda, \mu \in \sigma_{p}\left(\mathcal{A}_{p}^{*}\right) \\ \lambda \neq \mu}}|\lambda-\mu|>0
$$

Now we are ready to state and prove the main result of this section. A direct application of Ingham's Theorem $[10,13]$ yields the exact controllability of the boundary control system for any time $\tau>\frac{2 \pi}{\gamma_{0}}$ where $\gamma_{0}$ is the gap of the eigenvalues of $\mathcal{A}_{p}^{*}$ given by (4.6). However, in general this gap is less than that of the asymptotic distance between consecutive eigenvalues. To provide a smaller lower bound for the time of exact controllability we will separate the low and high frequencies as in Proposition 8.1.3 of [22]. However, we need to modify the arguments in the said proposition since the eigenvectors are not orthogonal anymore, i.e., we generalize the proposition in such a way that it is still true for the case where the orthonormal basis is replaced by a Riesz basis. 
Theorem 4.3. Suppose that $\beta \geq 0$. Then the boundary control system $(\mathcal{F}, \mathcal{G})$ is exactly controllable in time $\tau$, if $\tau>\tau^{*}:=\frac{2 \ell}{\sqrt{\alpha A_{e}}}$. That means, for any $z^{0}, z^{1} \in \mathcal{X}_{0}^{\perp}$ there exists $\left(p_{0}, p_{1}\right) \in L^{2}\left([0, \tau], \mathbb{C}^{2}\right)$ such that the weak solution $z \in C\left([0, \tau], \mathcal{X}_{0}^{\perp}\right)$ of the system (1.8) with initial conditions (1.10) and boundary conditions (4.1) satisfies $z(\tau)=z^{1}$.

Proof. The proof of the existence, uniqueness and regularity of the weak solution will be provided later (see Thm. 4.4 below). We divide the proof into several steps for ease of reading. Moreover, we first assume that $\beta \neq 2 \mu_{n}$ for all $n \in \mathbb{N}$.

Step 1. Let us prove that $\mathcal{B}$ is an admissible control operator for $\left(\mathrm{e}^{\mathcal{A}_{p} t}\right)_{t \geq 0}$, or equivalently, $\mathcal{B}^{*}$ is an admissible observation operator for the adjoint semigroup $\left(\mathrm{e}^{\mathcal{A}_{p}^{*} t}\right)_{t \geq 0}$. The latter means that for each $t \geq 0$ there exists $C_{t}>0$ such that $\int_{0}^{t}\left|\mathcal{B}^{*} \mathrm{e}^{*} \mathcal{A}_{p}^{*} z\right|^{2} \mathrm{~d} s \leq C_{t}\|z\|_{\mathcal{X}}^{2}$ for all $z \in \mathcal{D}\left(\mathcal{A}_{p}^{*}\right)$. According to (3.10), (3.11) and the asymptotic behavior of $\mu_{n}$ given in (3.9) we have $\frac{1}{\mu_{n}^{2}}\left\|z_{n}^{*}\right\|_{\mathcal{X}}^{2} \rightarrow \frac{A_{T}^{2} \ell}{\gamma^{2} \alpha A_{\mathrm{e}}^{2}}$ as $|n| \rightarrow \infty$. Using this, we can see that

$$
0<d_{y}:=\inf _{n \in \mathbb{Z}^{*}} \frac{\left|\psi_{n}(y)\right|^{2}}{\left\|z_{n}^{*}\right\|_{\mathcal{X}}^{2}} \leq \sup _{n \in \mathbb{Z}^{*}} \frac{\left|\psi_{n}(y)\right|^{2}}{\left\|z_{n}^{*}\right\|_{\mathcal{X}}^{2}}=: D_{y}<\infty, \quad y=0, \ell
$$

Let $M=M(\beta)$ the largest integer such that $\beta>2 \mu_{M}$. Thus $\lambda_{n}$ is real whenever $|n| \leq M$. From (3.16), Ingham's Theorem [10,13], (4.7) and (3.15) we have

$$
\begin{aligned}
\int_{0}^{t}\left|\mathcal{B}^{*} \mathrm{e}^{\mathcal{A}_{p}^{*} s} z\right|^{2} \mathrm{~d} s & =\sum_{y=0, \ell} \int_{0}^{t}\left|\sum_{n \in \mathbb{Z}^{*}} \mathrm{e}^{\lambda_{n} s}\left\langle z, \tilde{z}_{n}\right\rangle \mathcal{X} \frac{\psi_{n}(y)}{\left\|z_{n}^{*}\right\|_{\mathcal{X}}}\right|^{2} \mathrm{~d} s \\
& \leq 2 \sum_{y=0, \ell}\left(\int_{0}^{t} \sum_{0<|n| \leq M} \mathrm{e}^{2 \lambda_{n} s}\left|\left\langle z, \tilde{z}_{n}\right\rangle_{\mathcal{X}}\right|^{2} \frac{\left|\psi_{n}(y)\right|^{2}}{\left\|z_{n}^{*}\right\|_{\mathcal{X}}^{2}}+\left|\sum_{|n|>M} \mathrm{e}^{\left(-\beta / 2+i \Im \lambda_{n}\right) s}\left\langle z, \tilde{z}_{n}\right\rangle \mathcal{X} \frac{\psi_{n}(y)}{\left\|z_{n}^{*}\right\| \mathcal{X}}\right|^{2} \mathrm{~d} s\right) \\
& \leq C_{t} \sum_{y=0, \ell} \sum_{n \in \mathbb{Z}^{*}}\left|\left\langle z, \tilde{z}_{n}\right\rangle_{\mathcal{X}}\right|^{2} \frac{\left|\psi_{n}(y)\right|^{2}}{\left\|z_{n}^{*}\right\|_{\mathcal{X}}^{2}} \leq C_{t}\left(D_{0}+D_{\ell}\right) c^{-2}\|z\|_{\mathcal{X}}^{2}
\end{aligned}
$$

for all $z \in \mathcal{D}\left(\mathcal{A}_{p}^{*}\right)$ and $t \geq 0$. Hence $\mathcal{B}^{*}$ is an admissible observation operator for $\left(\mathrm{e}^{\mathcal{A}_{p}^{*} t}\right)_{t \geq 0}$.

Step 2. Now we separate the eigenfunctions into two parts. From (4.5), for arbitrary $\epsilon>0$, there exists a positive integer $N=N(\epsilon)$ such that $\Re \lambda_{n}=-\beta / 2$ for all $|n|>N$ and

$$
\inf _{\substack{|m|,|n|>N \\ m \neq n}}\left|\Im \lambda_{n}-\Im \lambda_{m}\right| \geq \frac{\sqrt{\alpha A_{e}}}{\ell} \pi-\epsilon .
$$

Consider the subspace $\mathcal{X}_{1}=\operatorname{span}\left(z_{n}^{*}\right)_{0<|n| \leq N}$ of $\mathcal{X}_{0}^{\perp}$. It is clear that $\left(z_{n}^{*}\right)_{0<|n| \leq N}$ is linearly independent and so it forms a basis for $\mathcal{X}_{1}$. For each $t \in \mathbb{R}$, let $T(t)^{*}$ be the restriction of $\mathrm{e}^{\mathcal{A}_{p}^{*} t}$ to $\mathcal{X}_{1}$. We note that by construction $\sigma\left(\mathcal{A}_{p}^{*} \mid \mathcal{X}_{1}\right)=\left(\lambda_{n}\right)_{0<|n| \leq N}$.

Let $\mathcal{X}_{2}=\operatorname{clos} \operatorname{span}\left(z_{n}^{*} /\left\|z_{n}^{*}\right\|_{\mathcal{X}}\right)_{|n|>N}$ and $\mathcal{A}_{q}^{*}$ be the part of $\mathcal{A}_{p}^{*}$ in $\mathcal{X}_{2}$. Notice that $\mathcal{A}_{q}^{*}$ have also compact resolvent and $\sigma\left(\mathcal{A}_{q}^{*}\right)=\sigma\left(\mathcal{A}_{p}^{*}\right) \backslash\left(\lambda_{n}\right)_{0<|n| \leq N}$. Since $\operatorname{span}\left(z_{n}^{*} /\left\|z_{n}^{*}\right\|_{\mathcal{X}}\right)_{|n|>N}$ is invariant under the $\mathcal{C}_{0}$-group generated by $\mathcal{A}_{p}^{*}$, its closure is also invariant under this $\mathcal{C}_{0}$-group. Thus $\mathcal{A}_{q}^{*}$ also generates a $\mathcal{C}_{0}$-group on $\mathcal{X}_{2}$ and $\left.\mathrm{e}^{\mathcal{A}_{p}^{*} t}\right|_{\mathcal{X}_{2}}=\mathrm{e}^{\mathcal{A}_{q}^{*} t}$ for all $t \in \mathbb{R}$.

Step 3. From the discussions in the previous step, we can see that the normalized eigenvectors $\left(z_{n}^{*} /\left\|z_{n}^{*}\right\|_{\mathcal{X}}\right)_{|n|>N}$ of $\mathcal{A}_{q}^{*}$ form a Riesz basis for $\mathcal{X}_{2}$. Let $\mathcal{B}_{q}^{*}$ be the restriction of $\mathcal{B}^{*}$ to $\mathcal{D}\left(\mathcal{A}_{q}^{*}\right)$. A similar application of Ingham's Theorem as above shows that $\mathcal{B}_{q}^{*}$ is an admissible observation operator for the semigroup generated by $\mathcal{A}_{q}^{*}$. 
Moreover, from Ingham's Theorem and (3.15) we have the inverse estimate

$$
\begin{aligned}
\int_{0}^{\tau}\left|\mathcal{B}_{q}^{*} \mathrm{e}^{\mathcal{A}_{q}^{*} t} z\right|^{2} \mathrm{~d} t & \geq \sum_{y=0, \ell} \mathrm{e}^{-\beta \tau} \int_{0}^{\tau}\left|\sum_{|n|>N} \mathrm{e}^{i \Im \lambda_{n} s}\left\langle z, \tilde{z}_{n}\right\rangle_{\mathcal{X}} \frac{\psi_{n}(y)}{\left\|z_{n}^{*}\right\|_{\mathcal{X}}}\right|^{2} \mathrm{~d} s \\
& \geq c_{\tau} \sum_{y=0, \ell} \sum_{|n|>N}\left|\left\langle z, \tilde{z}_{n}\right\rangle_{\mathcal{X}}\right|^{2} \frac{\left|\psi_{n}(y)\right|^{2}}{\left\|z_{n}^{*}\right\|_{\mathcal{X}}^{2}} \geq c_{\tau}\left(d_{0}+d_{\ell}\right) C^{-2}\|z\|_{\mathcal{X}}^{2}
\end{aligned}
$$

for every $z \in \mathcal{D}\left(\mathcal{A}_{q}^{*}\right)$ and $\tau>\frac{2 \pi \ell}{\sqrt{\alpha A_{e}} \pi-\epsilon \ell}$. Thus, the pair $\left(\mathcal{A}_{q}^{*}, \mathcal{B}_{q}^{*}\right)$ is exactly observable in time $\tau>\frac{2 \pi \ell}{\sqrt{\alpha A_{e}} \pi-\epsilon \ell}$.

Step 4. Because $\left.\mathcal{A}_{p}^{*}\right|_{\mathcal{X}_{1}} \in \mathcal{L}\left(\mathcal{X}_{1}\right) \simeq \mathcal{L}\left(\mathbb{C}^{2 N}\right),\left.\mathcal{B}^{*}\right|_{\mathcal{X}_{1}} \in \mathcal{L}\left(\mathcal{X}_{1}, \mathbb{C}^{2}\right) \simeq \mathcal{L}\left(\mathbb{C}^{2 N}, \mathbb{C}^{2}\right)$ and $\left.\mathcal{B}^{*}\right|_{\mathcal{X}_{1}} z_{n}^{*} \neq 0$ for every $0<|n| \leq N$, the Hautus test for finite-dimensional systems implies that $\left(\mathcal{A}_{p}^{*}\left|\mathcal{X}_{1}, \mathcal{B}^{*}\right| \mathcal{X}_{1}\right)$ is observable. Since $\sigma\left(\left.\mathcal{A}_{p}^{*}\right|_{\mathcal{X}_{1}}\right) \cap \sigma\left(\mathcal{A}_{q}^{*}\right)=\emptyset$, according to Proposition 6.4.2 of [22] (see also [21]), the pairs $\left(\mathcal{A}_{p}^{*}\left|\mathcal{X}_{1}, \mathcal{B}^{*}\right|_{\mathcal{X}_{1}}\right)$ and $\left(\mathcal{A}_{q}^{*}, \mathcal{B}_{q}^{*}\right)$ are simultaneously exactly observable, in other words, there exists a constant $\tilde{c}_{\tau}>0$ such that for all $(v, w) \in \mathcal{X}_{1} \times \mathcal{D}\left(\mathcal{A}_{q}^{*}\right)$ it holds that

$$
\int_{0}^{\tau}\left|\mathcal{B}^{*}\right|_{\mathcal{X}_{1}} T(t)^{*} v+\left.\mathcal{B}_{q}^{*} \mathrm{e}^{\mathcal{A}_{q}^{*} t} w\right|^{2} \mathrm{~d} t \geq \tilde{c}_{\tau}\left(\|v\|_{\mathcal{X}}^{2}+\|w\|_{\mathcal{X}}^{2}\right)
$$

for every $\tau>\frac{2 \pi \ell}{\sqrt{\alpha A_{e}} \pi-\epsilon \ell}$. For $k \geq N$ define the $k$ th truncation of $z \in \mathcal{D}\left(\mathcal{A}_{p}^{*}\right)$ by

$$
z^{k}=\sum_{0<|n| \leq k}\left\langle z, \tilde{z}_{n}\right\rangle \mathcal{X} \frac{z_{n}^{*}}{\left\|z_{n}^{*}\right\|_{\mathcal{X}}}
$$

Then $z^{k} \rightarrow z$ in $\mathcal{X}$. Since $z^{N} \in \mathcal{X}_{1}$ and $z^{k}-z^{N} \in \operatorname{span}\left(\frac{z_{n}^{*}}{\left\|z_{n}^{*}\right\|_{\mathcal{X}}}\right)_{|n|>N} \subset \mathcal{D}\left(\mathcal{A}_{q}^{*}\right)$ it follows from (3.15) and (4.9) that for any $k>N$ we have

$$
\begin{aligned}
& \int_{0}^{\tau}\left|\mathcal{B}^{*} \mathrm{e}^{\mathcal{A}_{p}^{*} t} z^{k}\right|^{2} \mathrm{~d} t=\int_{0}^{\tau}\left|\mathcal{B}^{*}\right| \mathcal{X}_{1} T(t)^{*} z^{N}+\left.\mathcal{B}_{q}^{*} \mathrm{e}^{*} \mathcal{A}_{q}^{*} t\left(z^{k}-z^{N}\right)\right|^{2} \mathrm{~d} t \\
& \geq \tilde{c}_{\tau}\left(\left\|z^{N}\right\|_{\mathcal{X}}^{2}+\left\|z^{k}-z^{N}\right\|_{\mathcal{X}}^{2}\right) \geq \tilde{c}_{\tau} c^{2} \sum_{0<|n| \leq k}\left|\left\langle z^{k}, \tilde{z}_{n}^{*}\right\rangle \mathcal{X}\right|^{2} \geq \tilde{c}_{\tau} c^{2} C^{-2}\left\|z^{k}\right\|_{\mathcal{X}}^{2}
\end{aligned}
$$

Because $\mathcal{B}^{*}$ is an admissible observation operator for the semigroup generated by $\mathcal{A}_{p}^{*}$, letting $k \rightarrow \infty$ in the last inequality we obtain the inverse estimate

$$
\int_{0}^{\tau}\left|\mathcal{B}^{*} \mathrm{e}^{\mathcal{A}_{p}^{*} t} z\right|^{2} \mathrm{~d} t \geq \tilde{c}_{\tau} c^{2} C^{-2}\|z\|_{\mathcal{X}}^{2}
$$

Therefore $\left(\mathcal{A}_{p}^{*}, \mathcal{B}^{*}\right)$ is exactly observable in time $\tau>\frac{2 \pi \ell}{\sqrt{\alpha A_{e}} \pi-\epsilon \ell}$, and since $\epsilon>0$ is arbitrary, this pair is exactly observable in time $\tau>\frac{2 \ell}{\sqrt{\alpha A_{e}}}$.

If $\beta=2 \mu_{n}$ for some $n \in \mathbb{N}$ then one applies the above argument to the closure of the span of the normalized eigenvectors of $\mathcal{A}_{p}^{*}$. Notice that $\mathcal{B}^{*} Z^{*} \neq 0$. Then the series representation (3.17) together with Haraux's Theorem ([13], Thm. 4.5), imply the exact observability in the state space $\mathcal{X}_{0}^{\perp}$ for any time $\tau>\tau^{*}$. In any case, the conclusion of the theorem now follows from the well known duality of exact controllability and exact observability (e.g. [22], Thm. 11.2.1).

Now we address the existence and uniqueness of weak solutions of (1.8) under the boundary conditions (4.1). Let $p_{0}, p_{1} \in L_{\text {loc }}^{2}([0, \infty), \mathbb{C})$ and $z_{0} \in \mathcal{X}_{0}^{\perp}$. Since $\mathcal{B}$ is an admissible control operator for the semigroup generated by $\mathcal{A}_{p}$, then using the variation of parameters formula, the function

$$
z(t)=\mathrm{e}^{\mathcal{A}_{p} t} z^{0}+\int_{0}^{t} \mathrm{e}^{\left(\mathcal{A}_{p}^{*}\right)^{*}(t-s)} \mathcal{B}\left(p_{0}(s), p_{1}(s)\right) \mathrm{d} s \text { in } \mathcal{D}\left(\mathcal{A}_{p}^{*}\right)^{\prime}
$$


is the unique function that satisfies $z \in C\left([0, \infty), \mathcal{X}_{0}^{\perp}\right)$ and

$$
z(t)-z(0)=\int_{0}^{t}\left(\left(\mathcal{A}_{p}^{*}\right)^{*} z(s)+\mathcal{B}\left(p_{0}(s), p_{1}(s)\right)\right) \mathrm{d} s
$$

for all $t \geq 0$ (cf. [22], Rem. 4.2.6). The integral is computed in $\mathcal{D}\left(\mathcal{A}_{p}^{*}\right)^{\prime}$. Therefore, for each $\zeta \in \mathcal{D}\left(\mathcal{A}_{p}^{*}\right)$ we have from $(4.2)$

$$
\langle z(t)-z(0), \zeta\rangle_{\mathcal{X}}=\int_{0}^{t}\left(\left\langle z(s), \mathcal{A}_{p}^{*} \zeta\right\rangle_{\mathcal{X}}+\left\langle\left(p_{0}(s), p_{1}(s)\right), \mathcal{B}^{*} \zeta\right\rangle_{\mathbb{C}^{2}}\right) \mathrm{d} s
$$

and using the definition of $\mathcal{B}^{*}$ provided in Theorem 4.2, we can see that the components of $z$ comprise the unique weak solution of (1.8) with boundary conditions (4.1).

Theorem 4.4. If $z_{0} \in \mathcal{X}_{0}^{\perp}$ and $p_{0}, p_{1} \in L_{\mathrm{loc}}^{2}([0, \infty), \mathbb{C})$ then (1.8), (1.10), (4.1) has a unique weak solution $z \in C\left([0, \infty), \mathcal{X}_{0}^{\perp}\right) \cap H_{\mathrm{loc}}^{1}\left((0, \infty), \mathcal{D}\left(\mathcal{A}_{p}^{*}\right)^{\prime}\right)$ and for every $T>0$ there exists $C=C(T)>0$ such that

$$
\|z\|_{H^{1}\left((0, T), \mathcal{D}\left(\mathcal{A}_{p}^{*}\right)^{\prime}\right)}+\|z\|_{C\left([0, T], \mathcal{X}_{0}^{\perp}\right)} \leq C\left(\left\|z^{0}\right\|_{\mathcal{X}}+\left\|\left(p_{0}, p_{1}\right)\right\|_{L^{2}\left((0, T), \mathbb{C}^{2}\right)}\right)
$$

for all $z^{0} \in \mathcal{X}_{0}^{\perp}$ and $\left(p_{0}, p_{1}\right) \in L^{2}\left((0, T), \mathbb{C}^{2}\right)$. Moreover, if $z^{0} \in \mathcal{Z}$ and $p_{0}, p_{1} \in H^{1}((0, T), \mathbb{C})$ satisfy the compatibility condition $\mathcal{G} z^{0}=\left(p_{0}(0), p_{1}(0)\right)$ then the solution $z$ is in $C([0, T], \mathcal{Z}) \cap C^{1}\left([0, T], \mathcal{X}_{0}^{\perp}\right)$.

Proof. The first statement was already explained above and the estimate (4.11) can be shown from (4.10), while the second statement is a direct application of Proposition 10.1.8 from [22].

Remark 4.5. As in the proof of Theorem 4.3, it can be shown that $\mathcal{B}^{*}$ is an admissible observation operator for the semigroup generated by $\mathcal{A}_{p}$. This implies the following: For any $z_{0} \in \mathcal{D}\left(\mathcal{A}_{p}\right)$ the solution of the (unforced) system satisfies

$$
\|u(\cdot, 0)\|_{L^{2}(0, T)}+\|u(\cdot, \ell)\|_{L^{2}(0, T)} \leq C_{T}\left\|z_{0}\right\|_{\mathcal{X}} .
$$

By a standard density argument, one can use this to define the traces $u(\cdot, 0), u(\cdot, \ell) \in L^{2}(0, T)$ for the solution corresponding to the initial state $z_{0} \in \mathcal{X}_{0}^{\perp}$. Note that these traces do not make sense by the usual trace theorem for Sobolev spaces because in general $u \in C\left([0, T], L^{2}(0, \ell)\right)$. This is sometimes referred as a hidden regularity property of solutions, see $[12,14,15]$.

The controllability result Theorem 4.3 still holds even if there is only one forcing function that is applied to either of the tanks. In this case, the control operator would be either the first or second component of $\mathcal{B}$ according to where the control pressure is applied. The results can be also extended for two tanks with different horizontal cross sections. Now let us consider the case where the forcing is applied only at the left tank. In this case, the boundary operator $\mathcal{G}_{0}: \mathcal{Z}_{0} \rightarrow \mathbb{C}$ is defined by $\mathcal{G}_{0}\left(A, u, h_{0}, h\right)=A(0)-\gamma h_{0}$, where $\mathcal{Z}_{0}:=\left\{\left(A, u, h_{0}, h\right) \in H^{1}(0, \ell)^{2} \times \mathbb{C}^{2}: A(\ell)=\gamma h\right\} \cap \mathcal{X}_{0}^{\perp}$ is the corresponding solution space.

Theorem 4.6. In the situation of Theorem 4.3, where $\mathcal{G}$ is replaced by $\mathcal{G}_{0}$, the pair $\left(\mathcal{A}_{p}, \mathcal{B}_{0}\right)$ is not approximately controllable for any time $0<\tau<\tau^{*}$, where $\mathcal{B}_{0}$ is the control operator associated with the boundary control system $\left(\mathcal{F}, \mathcal{G}_{0}\right)$. In particular, $\left(\mathcal{A}_{p}, \mathcal{B}_{0}\right)$ is not exactly controllable for any time $0<\tau<\tau^{*}$.

Proof. From (3.3), there exists a positive integer $M$ such that $\left|\frac{\tau^{*} \Im \lambda_{n+1}}{2 \pi}-n\right|<\frac{1}{4}$ whenever $n>M$. By symmetry of the eigenvalues we have $\left|\frac{\tau^{*} \Im \lambda_{n-1}}{2 \pi}-n\right|<\frac{1}{4}$ for all $n<-M$. Now according to the Generalized Kadec $\frac{1}{4}$ Theorem ([26], Cor. 2, p. 196), the system of exponentials $\left(\mathrm{e}^{i 2 n \pi t / \tau^{*}}\right)_{0 \leq|n| \leq M} \cup\left(\mathrm{e}^{\lambda_{n+1} t}\right)_{|n|>M}$ forms a Riesz basis for $L^{2}\left(0, \tau^{*}\right)$. Let $\left(g_{n}\right)_{n \in \mathbb{Z}}$ be the sequence biorthogonal to this Riesz basis. Given $0<\tau<\tau^{*}$, let us take a nonzero element $F_{1} \in L^{2}\left(0, \tau^{*}-\tau\right)$ such that

$$
\int_{0}^{\tau^{*}-\tau} F_{1}(t) \overline{g_{n}(\tau+t)} \mathrm{d} t=0, \quad 0 \leq|n| \leq M,
$$


that is, $F_{1}$ is in the orthogonal complement of the subspace of $L^{2}\left(0, \tau^{*}-\tau\right)$ spanned by the functions $\left(g_{n}(\tau+\cdot)\right)_{0 \leq|n| \leq M}$. Define the nonzero element $F \in L^{2}\left(0, \tau^{*}\right)$ by $F(t)=0$ if $0 \leq t \leq \tau$ and $F(t)=F_{1}(t-\tau)$ if $\tau<t \leq \tau^{*}=2 \ell / \sqrt{\alpha A_{e}}$.

Define

$$
z=\sum_{|n|>M}\left(\left\langle F, g_{n}\right\rangle_{L^{2}\left(0, \tau^{*}\right)} \frac{\left\|z_{n+1}^{*}\right\|_{\mathcal{X}}}{\psi_{n+1}(0)}\right) \frac{z_{n+1}^{*}}{\left\|z_{n+1}^{*}\right\|_{\mathcal{X}}} .
$$

This is a nonzero element of $\mathcal{X}_{0}^{\perp}$ because $\left(\left\langle F, g_{n}\right\rangle_{L^{2}\left(0, \tau^{*}\right)}\left\|z_{n+1}^{*}\right\|_{\mathcal{X}} \psi_{n+1}(0)^{-1}\right)_{|n|>M}$ is nonzero element in $\ell^{2}$. Note that by the uniqueness of the coefficients in a series of the elements of the Riesz basis, we must have $\left\langle F, g_{n}\right\rangle_{L^{2}\left(0, \tau^{*}\right)}\left\|z_{n+1}^{*}\right\|_{\mathcal{X}} \psi_{n+1}(0)^{-1}=\left\langle z, \tilde{z}_{n+1}\right\rangle_{\mathcal{X}}$ for all $|n|>M$ and so

$$
\mathcal{B}_{0}^{*} \mathrm{e}^{\mathcal{A}_{p}^{*} t} z=\sum_{|n|>M} \mathrm{e}^{\lambda_{n+1} t}\left\langle z, \tilde{z}_{n+1}\right\rangle_{\mathcal{X}} \frac{\psi_{n+1}(0)}{\left\|z_{n+1}^{*}\right\| \mathcal{X}}=\sum_{|n|>M}\left\langle F, g_{n}\right\rangle_{L^{2}\left(0, \tau^{*}\right)} \mathrm{e}^{\lambda_{n+1} t}=F(t) .
$$

The terms with indices $0 \leq|n| \leq M$ vanish by construction of $F$. Hence there exists $z \in \mathcal{X}_{0}^{\perp} \backslash\{0\}$ such that $\mathcal{B}_{0}^{*} \mathrm{e}^{\mathcal{A}_{p}^{*}(\cdot)} z=0$ in $L^{2}(0, \tau)$. Therefore $\mathcal{N}\left(\mathcal{B}_{0}^{*} \mathrm{e}^{\mathcal{A}_{p}^{*}(\cdot)}\right) \neq\{0\}$ so that the adjoint system $\left(\mathcal{A}_{p}^{*}, \mathcal{B}_{0}^{*}\right)$ is not approximately observable in time $\tau$ for any $0<\tau<\tau^{*}$. The theorem follows from the duality of approximate observability and approximate controllability.

\section{System With Distributed CONTROL}

One could also consider external control pressure applied to a part of the elastic tube, e.g. $[2,18,23]$. In this case, the linearized momentum equation becomes

$$
\frac{\partial u}{\partial t}=-\alpha \frac{\partial A}{\partial x}-\beta u+P_{c} \chi_{[a, b]}
$$

where $P_{c} \in L_{\text {loc }}^{2}\left([0, \infty), L^{2}(0, \ell)\right), 0<a<b<\ell$. In the literature, the control has to vanish at the endpoints of the subinterval $[a, b]$ where it is applied, however, we consider the general case where this vanishing condition is not assumed.

In the present situation, the control operator $\mathcal{B}_{1}: L^{2}(0, \ell) \rightarrow \mathcal{X}_{0}^{\perp}$ is bounded and given by $\mathcal{B}_{1} P_{c}=$ $\left(0, P_{c} \chi_{[a, b]}, 0,0\right)$. For each $z=\left(A, u, h_{0}, h\right) \in \mathcal{X}_{0}^{\perp}$ and $P_{c} \in L^{2}(0, \ell)$ we have $\left\langle\mathcal{B}_{1} P_{c}, z\right\rangle_{\mathcal{X}}=\frac{1}{\alpha}\left\langle P_{c}, u \chi_{[a, b]}\right\rangle_{L^{2}(0, \ell)}$. Thus, the operator $\mathcal{B}_{1}^{*}: \mathcal{X}_{0}^{\perp} \rightarrow L^{2}(0, \ell)$ is given by $\mathcal{B}_{1}^{*}\left(A, u, h_{0}, h\right)=\frac{1}{\alpha} u \chi_{[a, b]}$. We have the following result, whose proof is similar as in the previous section, and hence it is omitted.

Theorem 5.1. The pair $\left(\mathcal{A}_{p}, \mathcal{B}_{1}\right)$ is exactly controllable in time $\tau$ if $\tau>\frac{2 \ell}{\sqrt{\alpha A_{e}}}$.

\section{APPENDix}

We prove the equivalence of (2.2) and (2.3) stated in the proof of the well-posedness in Section 2. Notice that $(2.2)$ is the system of equations

$$
\left\{\begin{aligned}
\lambda A+A_{e} u_{x} & =B \\
(\lambda+\beta) u+\alpha A_{x} & =v \\
\lambda h_{0}+\frac{A_{e}}{A_{T}} u(0) & =g_{0} \\
\lambda h-\frac{A_{e}}{A_{T}} u(\ell) & =g .
\end{aligned}\right.
$$

Suppose that (2.2), and hence (A.1), has a solution $\left(A, u, h_{0}, h\right)$ in $\mathcal{D}(\mathcal{A})$. Multiplying the first equation in (A.1) by $\frac{\gamma}{A_{T}} \bar{\psi}$ for $\psi \in H^{1}(0, \ell)$, integrating by parts, solving for $u$ in the second equation of (A.1) and using the boundary conditions we obtain (2.3). 
Conversely, let $A \in H^{1}(0, \ell)$ satisfy (2.3) for all $\psi \in H^{1}(0, \ell)$. Define

$$
\begin{aligned}
u & =\frac{1}{\lambda+\beta}\left(v-\alpha A_{x}\right), \\
h_{0} & =\frac{1}{\lambda}\left(g_{0}-\frac{A_{e}}{A_{T}} u(0)\right), \\
h & =\frac{1}{\lambda}\left(g+\frac{A_{e}}{A_{T}} u(\ell)\right) .
\end{aligned}
$$

Notice that $u$ solves the second equation of (A.1) while (A.3) and (A.4) are the third and fourth. From (2.3) and (A.2) we have

$$
\begin{aligned}
\frac{\gamma}{A_{T}} \int_{0}^{\ell}(\lambda A(x)-B(x)) \overline{\psi(x)} \mathrm{d} x= & \frac{\gamma}{A_{T}} \int_{0}^{\ell} A_{e} u(x) \overline{\psi_{x}(x)} \mathrm{d} x+\left(\gamma g_{0}-\lambda A(0)\right) \overline{\psi(0)} \\
& +(\gamma g-\lambda A(\ell) \overline{\psi(\ell) .}
\end{aligned}
$$

Since the above equation is true for all $\psi \in H^{1}(0, \ell)$, it also holds in particular for all test functions $\psi \in C_{0}^{\infty}(0, \ell)$, and so the above equation gives us

$$
\int_{0}^{\ell}\left(B(x)-\lambda A(x) \overline{\psi(x)} \mathrm{d} x=-\int_{0}^{\ell} A_{e} u(x) \overline{\psi_{x}(x)} \mathrm{d} x, \quad \forall \psi \in C_{0}^{\infty}(0, \ell),\right.
$$

which implies that $B-\lambda A=\left(A_{e} u\right)_{x}$ or $u_{x}=\frac{1}{A_{e}}(B-\lambda A) \in L^{2}(0, \ell)$. As a consequence, $u \in H^{1}(0, \ell)$ and the first equation in (A.1) holds. It remains to verify the boundary conditions $A(0)=\gamma h_{0}$ and $A(\ell)=\gamma h$. The left hand side of (A.5) can be written as

$$
\begin{aligned}
& \frac{\gamma}{A_{T}} \int_{0}^{\ell}\left(\lambda A(x)-B(x) \overline{\psi(x)} \mathrm{d} x=-\frac{\gamma A_{e}}{A_{T}} \int_{0}^{\ell} u_{x}(x) \overline{\psi(x)} \mathrm{d} x\right. \\
& \quad=\frac{\gamma A_{e}}{A_{T}}(u(0) \overline{\psi(0)}-u(\ell) \overline{\psi(\ell)})+\frac{\gamma A_{e}}{A_{T}} \int_{0}^{\ell} u(x) \overline{\psi_{x}(x)} \mathrm{d} x
\end{aligned}
$$

and therefore, upon using (A.3)-(A.5) and the fact that $\lambda>0$,

$$
\left(\gamma h_{0}-A(0)\right) \overline{\psi(0)}+(\gamma h-A(\ell)) \overline{\psi(\ell)}=0
$$

for all $\psi \in H^{1}(0, \ell)$. Choosing appropriate functions $\psi$, this equation implies that $A(0)=\gamma h_{0}$ and $A(\ell)=\gamma h$. Therefore $\left(A, u, h_{0}, h\right) \in \mathcal{D}(\mathcal{A})$ and (A.1) holds.

Acknowledgements. The first author is supported by the grant Technologiestipendien Südostasien in the frame of the ASEA-Uninet granted by the Austrian Agency for International Cooperation in Education and Research (OeAD-GmbH), financed by the Austrian Federal Ministry for Science and Research (BMWF).

\section{REFERENCES}

[1] A. Bensoussan, G. Da Prato, M.C. Delfour and S.K. Mitter, Representation and Control of Infinite Dimensional Systems, 2nd ed. Birkhäuser, Boston (2007).

[2] A. Borzì and G. Propst, Numerical investigation of the Liebau phenomenon. Z. Angew. Math. Phys. 54 (2003) 1050-1072.

[3] W. Desch, E. Fas̆angová, J. Milota and G. Propst, Stabilization through viscoelastic boundary damping: a semigroup approach. Semigroup Forum 80 (2010) 405-415.

[4] K.J. Engel and R. Nagel, One-Parameter Semigroups for Linear Evolution Equations. Springer-Verlag, New York (2000). 
[5] M.A. Fernández, V. Milišić and A. Quarteroni, Analysis of a geometrical multiscale blood flow model based on the coupling of ODEs and Hyperbolic PDEs. Multiscale Model. Simul. 4 (2005) 215-236.

[6] B. Guo, Riesz basis approach to the stabilization of a flexible beam with a tip mass. SIAM J. Control Optim. 39 (2001) $1736-1747$.

[7] B. Guo, Riesz basis property and exponential stability of controlled Euler-Bernoulli beam equations with variable coefficients. SIAM J. Control Optim. 40 (2002) 1905-1923.

[8] B. Guo, J.M. Wang and S.P. Wang, On the $C_{0}$-semigroup generation and exponential stabilization resulting from a shear force feedback on a rotating beam. Syst. Control Lett. 54 (2005) 557-574.

[9] B. Guo and R. Yu, The Riesz basis property of discrete operators and applications to a Euler-Bernoulli beam equations with boundary linear feedback control. IMA J. Math. Control Inf. 18 (2001) 241-251.

[10] A.E. Ingham, Some trigonometrical inequalities with applications to the theory of series. Math. Z. 41 (1936) $367-379$.

[11] B. Jacob and H. Zwart, Linear Port Hamiltonian Systems on Infinite-Dimensional Spaces. Birkhäuser, Basel (2012).

[12] V. Komornik, Exact Controllability and Stabilization: the Multiplier Method. Wiley-Masson, Paris-Chicester (1994).

[13] V. Komornik and P. Loreti, Fourier Series in Control Theory. Springer-Verlag, New York (2005).

[14] I. Lasiecka and R. Triggiani, Regularity of hyperbolic equations under $L^{2}\left(0, T ; L^{2}(\Gamma)\right)$ boundary terms. Appl. Math. Optim. 10 (1983) 275-286

[15] J.-L. Lions, Contrôle des systèmes distribués singuliers. Gauthiers-Villars, Paris (1968).

[16] M. Miklavčič, Applied Functional Analysis and Partial Differential Equations. World Scientific, Singapore (1998).

[17] J.T. Ottesen, Valveless pumping in a fluid-filled closed elastic tube-system: one-dimensional theory with experimental validation. J. Math. Biol. 46 (2003) 309-332.

[18] H.J. Rath and I. Teipel, Der Fördereffekt in ventillosen, elastischen Leitungen. Z. Angew. Math. Phys. 29 (1978) 123-133.

[19] W. Ruan, A coupled system of ODEs and quasilinear hyperbolic PDEs arising in a multiscale blood flow model. J. Math. Anal. Appl. 343 (2008) 778-798.

[20] O. Staffans, Well-Posed Linear Systems. Cambridge University Press (2005).

[21] M. Tucsnak and G. Weiss, Simultaneous exact controllability and some applications. SIAM J. Control Optim. 38 (2000) $1408-1427$.

[22] M. Tucsnak and G. Weiss, Observation and Control for Operator Semigroups. Birkhäuser-Verlag, Basel (2009).

[23] H.J. von Bredow, Untersuchung eines ventillosen Pumpprinzips. Fortschr. Ber. VDI-Zeitschr. Reihe 7 (1968).

[24] G. Xu and B Guo, Riesz basis property of evolution equations in Hilbert spaces and application to a coupled string equation. SIAM J. Control Optim. 42 (2003) 966-984.

[25] C.-Z. Xu and G. Weiss, Eigenvalues and eigenvectors of semigroup generators obtained from diagonal generators by feedback. Commun. Inf. Syst. 11 (2011) 71-104.

[26] R. Young, An Introduction to Nonharmonic Fourier Analysis. Academic Press, New York (1980). 